TITLE: RECENT DEVELOPMENTS IN NEUTRINO PHYSICS

Author(s): Gerald T. Garvey

SUBMITTED TO: Proceedings of the EPS Conference on Hadronic Structure and Electroweak Interactions, amsterdam, 5-10 August 1991, (to be Published as a special issue of the Journal Nuclear Physics A.

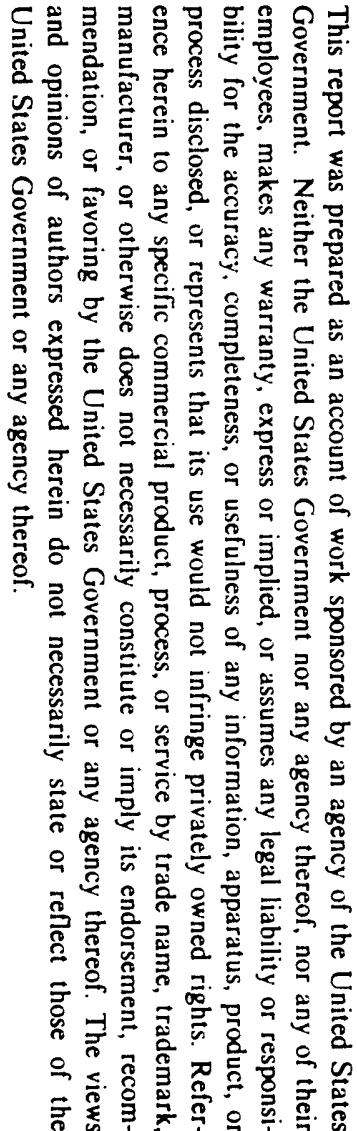




\section{Recent developments in neutrino physics}

G. T. Garvey

Los Alamos National Laboratory, MP-4, MS H846, Los Alamos, New Mexico 87545, USA

\section{INTRODUCTION}

I shall attempt to summarize recent developments in the experimental situation in neutrino physics. The paper will deal with recent results, drawing on either published work or research that has been presented in preprint form, as there is an adequate supply of interesting and controversial data restricting oneself to these generally more reliable sources. The discussion of the theoretical implication of these experimental results will be presented in the following paper by Boris Kayser.

The topics to be covered in this presentation are:

(1) direct measurements of $\bar{\nu}_{e}$ mass via beta endpoint studies;

(2) status of solar neutrino observations;

(3) status of " $17-\mathrm{keV}$ neutrino" reports; and

(4) the use of $\nu p$ elastic scattering to determine the "strange quark" content of the proton.

Before launching into these topics, attention should be called to the new result from LEP. Dydak [1] reported earlier this week that the number of standard model type neutrinos that couple to the $Z_{0}$ is now known to be $2.95 \pm 0.05$. Thus, with ever increasing precision the LEP program verifies the status of minimum standard model (MSM); on the other hand, there is strong suggestion in the research presented below that extensions to the minimal standard model are required, to allow for both neutrino mass and mixing.

\section{DIRECT $\bar{\nu}_{e}$ MEASUREMENTS}

To begin, let us consider the direct measurement of the $\bar{\nu}_{e}$ mass via examination of the endpoint of ${ }^{3} \mathrm{H}$ decay,

${ }^{3} \mathrm{H} \rightarrow{ }^{3} \mathrm{He}+\bar{\nu}_{e}+e^{-}+18.6 \mathrm{keV}$.

The normal beta spectrum $\left(m_{\bar{\nu}_{e}}=0\right)$

$N\left(E_{e}\right)=C F\left(Z, R, E_{e}\right) p_{e} E_{e}\left(E_{0}-E\right)^{2}$ 
is modified if the $\bar{\nu}_{e}$ has a mass

$N\left(E_{e}\right)=C F\left(Z, R . E_{e}\right) p_{e} E_{e}\left(E_{0}-E\right)^{2}\left(1-\frac{m_{\nu}^{2}}{\left(E-E_{0}\right)^{2}}\right)^{\frac{1}{2}}$.

where $C$ is a normalization constant and $F\left(Z, R, E_{e}\right)$ is the Coulomb Fermi function. Once $E-E_{0} \gg m_{\nu}$, the spectrum is, for all intents and purposes, identical with Eq. (2). The modification to the spectrum occurs in the vicinity of a few times $m_{\nu}$ below the endpoint. The situation in the real case is even more complicated because the undetected electron is not necessarily in its ground state around ${ }^{3} \mathrm{He}$ so that a more proper characterization of the beta spectrum due to the final-state spectrum (FSS) of the ${ }^{3} \mathrm{He}^{+}$ion is

$\mathcal{N}\left(E_{e}\right)=C F\left(Z, R, E_{0}\right) p_{e} E_{e} \sum_{i} w_{i}\left(E_{0}-E_{i}-E_{e}\right)\left(E_{0}-E_{i}-E_{e}-m_{\nu}^{2}\right)^{1 / 2}$

where $E_{0}$ is the endpoint energy with the valence electron in the ${ }^{3} \mathrm{He}^{+}$ground state. $E_{i}$ is the excitation energy of the electron, and $w_{i}$ the probability of the excitation. As $E_{i}$ for the $1 s-2 p$ excitation is some $25 \mathrm{eV}$, it is essential to know the final-state spectrum accurately to extract a proper value of $m_{\bar{\nu}_{e}}$. Figure 1 shows how large these effects are. In the case of ${ }^{3} \mathrm{H}_{2}$, only $60 \%$ of the time is the unobserved electron in the ground state.

These measurements came to prominence when in 1980 the ITEP group announced [2] that they had measured an electron antineutrino mass of some $30 \mathrm{eV}$. They have persisted [3] in claiming a finite $\left(17<m_{\bar{\nu}_{\mathrm{e}}} c^{2}<40 \mathrm{eV}\right)$ neutrino mass.

As experiments sensitive to this small a value of the neutrino mass are very difficult to set up, deal with the specific backgrounds, and investigate systematic errors, results from the first round of experiments following the ITEP measurements did not occur until 1986-87. These results are listed in Table 1. While no experiment found a positive result for neutrino mass, the limits obtained did not rule out the lowest range reported by ITEP.

The next generation of these experiments is now being completed. The published resuit from the INS-Tokyo group [4] is

$m_{\bar{\nu}_{e}} c^{2}<13 \mathrm{eV} \quad 95 \%$ C.L.

They have carried out a high-statistics experiment $\left(150 \mathrm{~K}\right.$ events $\left(E_{0}-E_{\mathrm{e}}\right)<100 \mathrm{eV}$ ) using arachiatic acid as a source which, while complicated, allows them to place ${ }^{109} \mathrm{Cd}$ in the compound. ${ }^{109} \mathrm{Cd}$ has a conversion line at $18.511 .7 \pm 1.3 \mathrm{eV}$, which is excellent for calibration purposes and allows them to obtain a precise knowledge of their response function. They attribute a $65-\mathrm{eV}^{2}$ systematic uncertainty to their knowledge of the FSS for an arachiatic acid source, but with our present state of understanding, it is difficult to assess if this is adequate. 


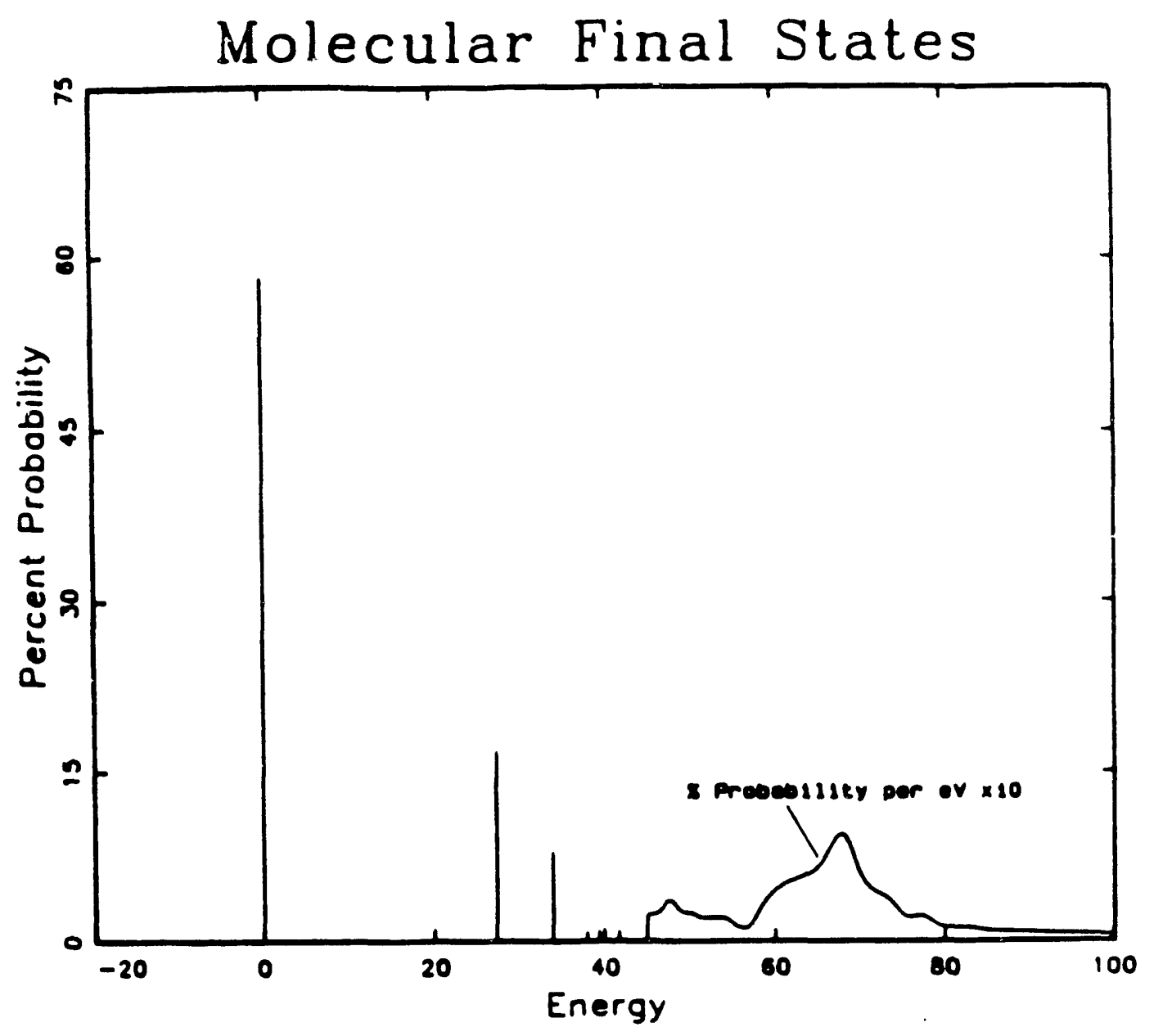

Figure 1. Probability for excitation of the unobserved electron for the decay of molecular ${ }^{3} \mathrm{H}$.

Table 1

List of the results from the first generation experiments following the ITEP [2] positive results

\begin{tabular}{llc}
\hline Group & Source & $\begin{array}{c}\text { Result }\left(\mathrm{eV} / \mathrm{c}^{2}\right) \\
95 \% \text { C.L. }\end{array}$ \\
\hline Zürich $^{(a)}$ & ${ }^{3} \mathrm{H}$ in graphite & $<18$ \\
LANL $^{(b)}$ & ${ }^{3} \mathrm{H}_{2}$ gas & $<27$ \\
INS-Tokyo $^{(c)}$ & arachiatic acid & $<32$ \\
\hline
\end{tabular}

(a) M. Fritschisset et al., Phys. Lett. B173 (1986) 485.

(b) J. Wilkerson et al., Phys. Rev. Lett. 58 (1987) 2023.

(c) H. Kawakami et al., Phys. Lett. B187 (1987) 198. 
A preprint [5] from the Los Alamos group reporting on somewhat lower statistics (20K events, $\left.\left(E_{0}-E_{e}\right)<100 \mathrm{eV}\right)$, an experiment which employs a ${ }^{3} \mathrm{H}_{2}$ gaseous source finds a limit of

$m_{\bar{\nu}_{e}} c^{2}<9.3 \mathrm{eV} \quad 95 \%$ C.L.

Their reported energy resolution is $16.2 \mathrm{eV}$ with the calibration and response measured via a ${ }^{83} \mathrm{Kr}(17820.1 \pm 18 \mathrm{eV})$ decay. The ${ }^{83} \mathrm{Kr}$ is easily incorporated into their ${ }^{3} \mathrm{H}_{2}$ source. It is interesting and impressive that $93.5 \%$ of the ${ }^{83} \mathrm{Kr}$ conversion electrons escape without any energy loss. A major strength of this experiment, however, is that for ${ }^{3} \mathrm{H}_{2}$ the FSS can be calculated. There are three calculations [6-8] of $w_{i}\left(E_{0}-E_{i}\right)$ that agree to within $10 \mathrm{eV}^{2}$ on their effect on the extracted value of $m_{\bar{\nu}_{e}} c^{2}$. The physics approximations used in the calculations (Bohn-Oppenheimer and sudden approximation) are reckoned to have much smaller uncertainty $\left(0.04 \mathrm{eV}^{2}\right.$ and $2 \mathrm{eV}^{2}$, respectively).

Figures 2(a) and 2(b) show the residual errors after subtraction of the theoretical spectrum shape from the LANL measurement, assuming $m_{\bar{\nu}_{e}} c^{2}=30 \mathrm{eV}$ and $0 \mathrm{eV}$. respectively.
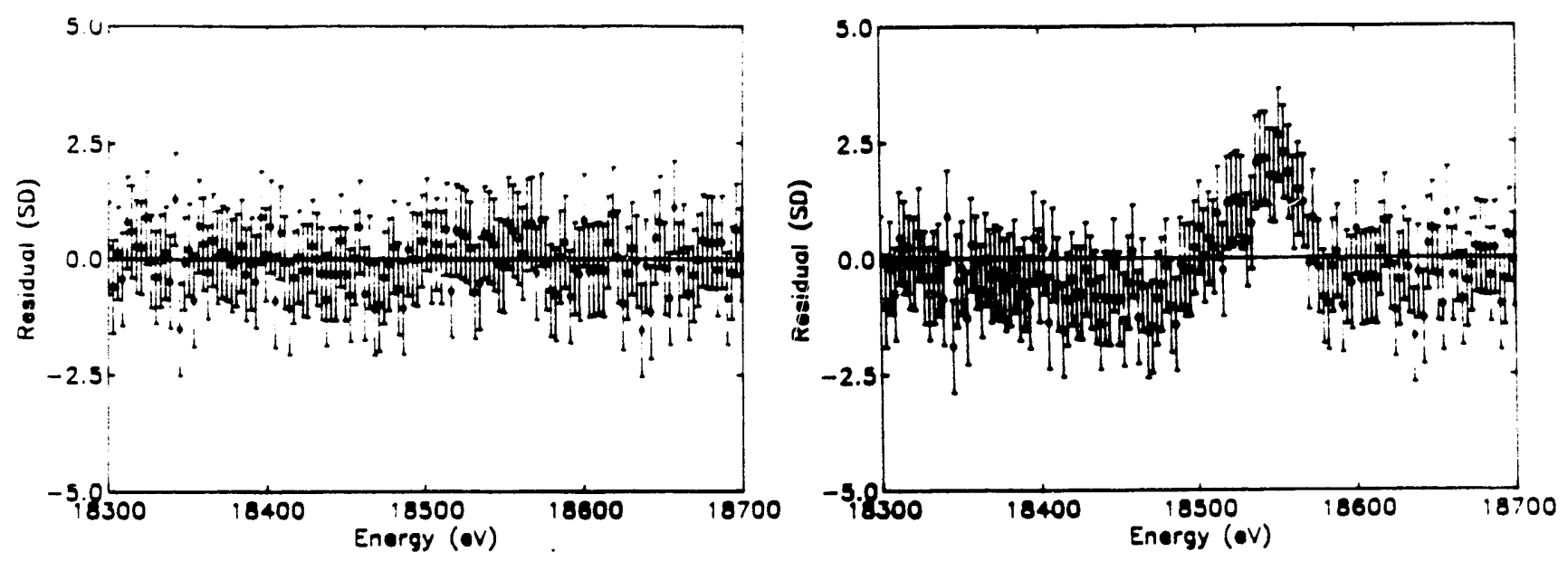

Figure 2. (a) Residuals (experiment-calculation), assuming $m_{\nu} c^{2}=0$; (b) residuals (experiment-calculation), assuming $m_{\nu} c^{2}=30 \mathrm{eV}$.

As Eq. (4) shows, the quantity directly extracted from the experiment is $m_{\bar{\nu}_{e}}$. The values from the two experiments are

$$
\begin{array}{lll}
m_{\nu}^{2} c^{4}=-65 \pm 85 \pm 65 \mathrm{eV}^{2} & \text { INS-Tokyo } & \text { and } \\
m_{\nu}^{2} c^{4}=-147 \pm 68 \pm 41 \mathrm{eV}^{2} & \text { LANL }
\end{array}
$$

Note both have a negative result for the most likely value of $m_{\nu}^{2} c^{4}$, which, of course, is not physically plausible. If this is not a statistical fluke, it may reflect a common undetected systematic error in these experiments. In both cases, the value for the limit 
on $m_{\bar{\nu}} c^{2}$ is fixed by renormalizing the fraction of reduced uncertainty for $m_{\frac{2}{\nu_{c}}} c^{4} \geq 0$ to unity.

It is clear that these experiments find no evidence for a neutrino mass well below the values claimed in [2] and [3]. Thus, positive evidence of a detectable mass for the electron neutrino has disappeared, with limit having been dropped by nearly an order of magnitude from where it was previous to the ITEP experiments.

There are third-generation experiments in this field that will be pressing into the regime of $m_{\nu}<3 \mathrm{eV}$. There are three different institutions involved in this round of experiments-LLNL in the US, INR-Moscow, and the University of Mainz in Germany. These are very large undertakings and they have many features in common. Figure 3 shows a schematic diagram of the Mainz setup. In common with the other three experiments, it has large acceptance achieved via magnetic transport, and extremely good energy resolution (2-3 eV) achieved via electrostatic means. The major problem in all these setups is background, as the signal rapidly diminishes in the vicinity of the endpoint. The last $10 \mathrm{eV}$ contains only $3 \times 10^{-10}$ of the total decay rate, so a sensitivity below $1 \mathrm{eV}$ will be likely impossible. Each group feels that the background problems are under control and that counting and analysis will soon begin. The LLNL group is using gaseous ${ }^{3} \mathrm{H}_{2}$, the Mainz group frozen ${ }^{3} \mathrm{H}_{2}$, and I don't know what the INR plans to use as their ${ }^{3} \mathrm{H}$ source. Results can be expected over the next few years from these groups, but the experiments are getting harder and harder. It is also evident that work is needed on the issue of FSS for whatever source is used for the tritium. The ${ }^{3} \mathrm{H}_{2}$ source used by the LANL group has a claimed systematic error of $10 \mathrm{eV}^{2}$ on the value

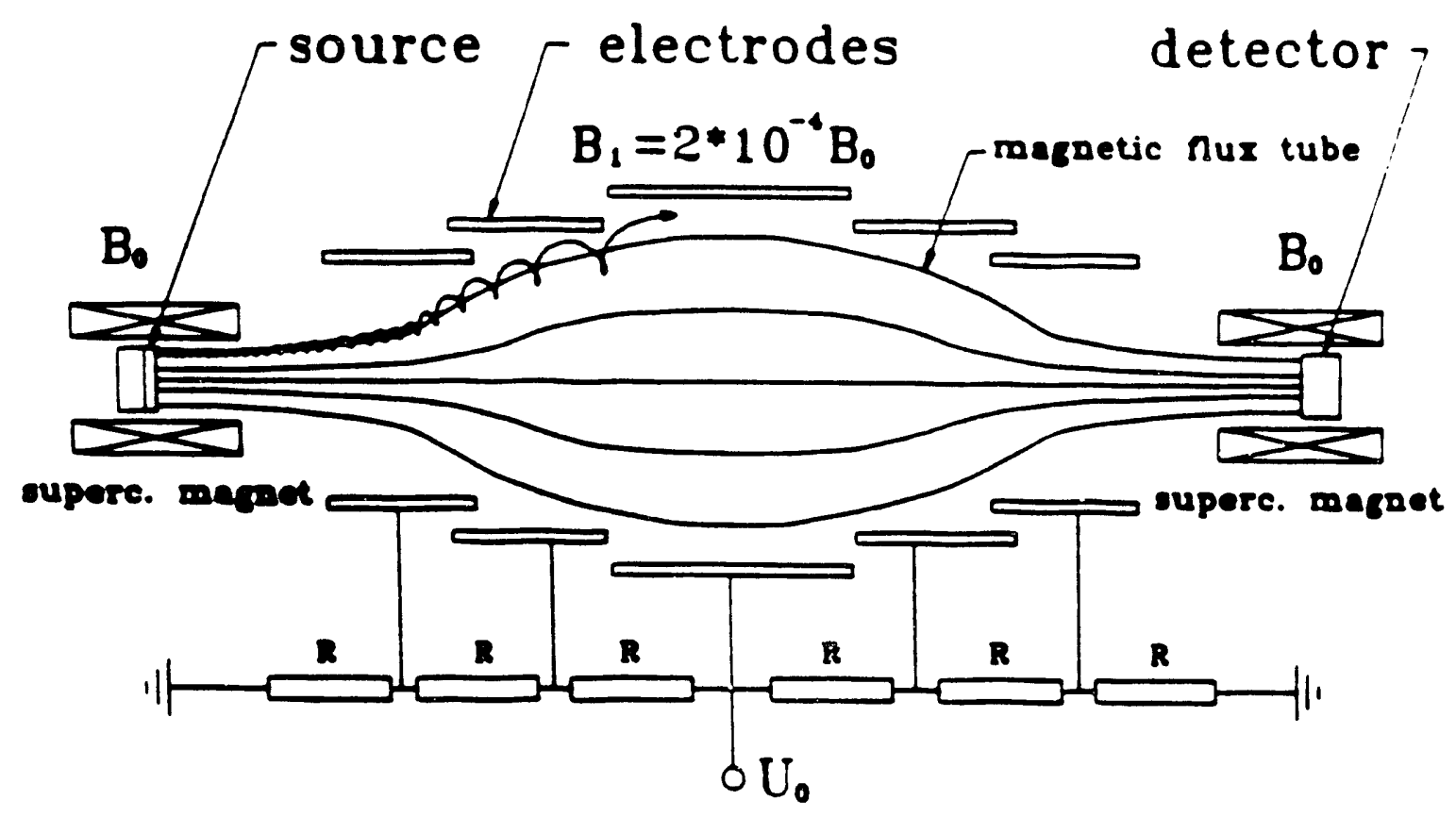

Figure 3. Schematic of the University of Mainz spectrometer for studying the ${ }^{3} \mathrm{H}$ endpoint. 
of $m \frac{2}{\nu_{e}}$ and so we will need improved calculations, or else atomic ${ }^{3} \mathrm{H}$ sources will have t) be used. There is a possibility that the energy resolution will be so good in these third-generation experiments and the statistics so high that one need only use the last $25 \mathrm{el}$ below the endpoint. which should be free of FSS effects.

\section{SOLAR NEUTRINO EXPERIMENTS}

The solar $v$ situation has not changed a great deal during the past six months. The SAGE group has come forth with a result in preprint form, which will be referred to after some background is provided. The sun produces the vast majority of its neutrinos in the process of converting $\mathrm{H}$ to ${ }^{4} \mathrm{He}$ [9]. Table 2 shows the principal reactions, and Table 3 lists the properties of the neutrino emitted in various stages of this process. Figure 4 shows the expected solar $\nu$ flux at the earth, based on the standard solar model. From Fig. 4 it is clear that the preponderance of high energy $\left(E_{\nu_{\mathrm{e}}}>2 \mathrm{MeV}\right)$ neutrinos comes from the decay of ${ }^{8} \mathrm{~B}$. This is a small branch $(0.02 \%)$ in the overall process of $4 p \rightarrow \alpha+2 e^{+}+2 \nu_{e}+26.7 \mathrm{MeV}$. As the Coulomb barriers for ${ }^{7} \mathrm{Be}$ and ${ }^{8} \mathrm{~B}$ formation are the highest in this process, the amount of ${ }^{8} \mathrm{~B}$ actually formed is extremely sensitive $\left(T^{18}\right)$ to the temperature at the center of the sun. There can be legitimate question about how well that temperature is known; a few percent reduction in its value leads to a factor of 2 reduction in the rate of ${ }^{8} \mathrm{~B}$ formation. This is not at all the case for the process $p+p \rightarrow d+e^{+}+\nu_{e}$, which is the principal energy source in the sun. Thus, if equilibrium is assumed between nuclear energy generation and the solar luminosity, the $\nu$ flux from the $p+p$ reaction is fixed to within $2 \%$.

As a neutrino source, the sun is unique because it is much farther away than any earth-based sources and insofar as the standard solar model is correct, we know what fluxes to expect. The great distance to the sun is important with regard to the possible phenomena of neutrino oscillations [10].

If we assume that the neutrinos $\nu_{e}$ and $\nu_{\mu}$ emitted in weak processes are actually a superposition of mass eigenstates ( 2 are assumed for simplicity)

$$
\begin{aligned}
& \left|\nu_{e}\right\rangle=\cos \theta_{v}\left|\nu_{1}\right\rangle+\sin \theta_{v}\left|\nu_{2}\right\rangle \\
& \left|\nu_{\mu}\right\rangle=-\sin \theta_{v}\left|\nu_{1}\right\rangle+\cos \theta_{v}\left|\nu_{2}\right\rangle,
\end{aligned}
$$

where $\nu_{1}$ and $\nu_{2}$ have masses $m_{1}$ and $m_{2}$ and $\cos \theta_{v}$ is the amplitude of $\nu_{1}$ in the electron neutrino when it decays in the absence of other matter (i.e., in vacuum). As these neutrinos propagate in free space, their amplitudes change as

$$
\begin{aligned}
\left|\nu_{e}(t)\right\rangle & =\cos \theta_{v} e^{i\left(E_{1} t-\mathbf{p} \cdot \mathbf{r}\right)}\left|\nu_{1}\right\rangle+\sin \theta_{v} e^{i\left(E_{2}-\mathbf{p} \cdot \mathbf{r}\right)}\left|\nu_{2}\right\rangle \\
& =\cos \theta_{v} e^{i\left(m_{1} / 2 p\right)^{2} t}\left|\nu_{1}\right\rangle+\sin \theta_{v} e^{i\left(m_{2} / 2 p\right)^{2} t}\left|\nu_{2}\right\rangle
\end{aligned}
$$

hence, after a time $t$, the original $\nu_{e}$ has altered its state so that

$\left|\left\langle\nu_{e} \mid \nu_{e}(t)\right\rangle\right|^{2}=1-\sin ^{2} 2 \theta_{v} \sin ^{2} \frac{\pi r}{L_{v}}$, 
Table 2

The reactions in the sun leading to the conversion of four protons into an alpha particle plus two positrons and electron neutrinos

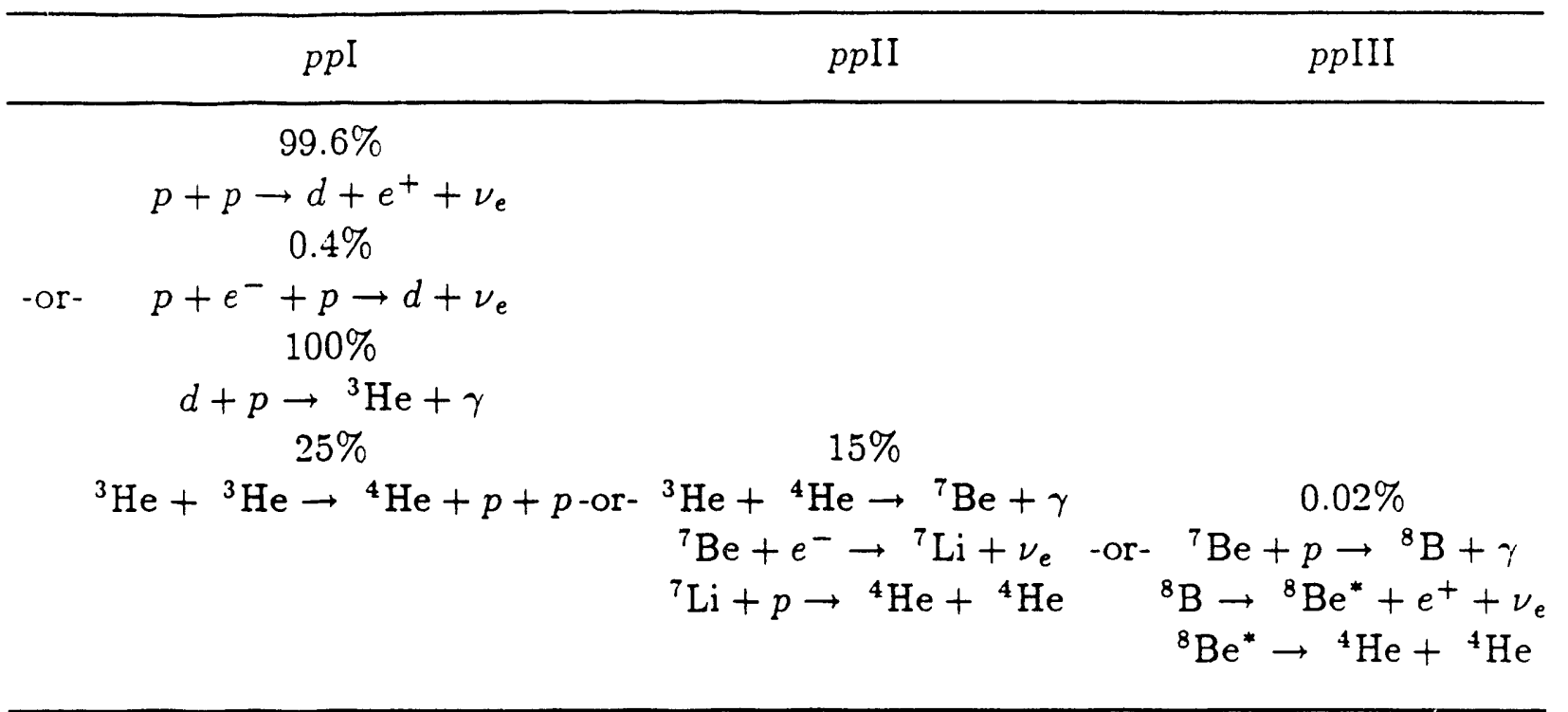

Table 3

The neutrino energy and flux from the principal weak interaction processes in the sun

\begin{tabular}{lcc}
\hline \multicolumn{1}{c}{ Solar Reaction } & $\begin{array}{r}\text { Maximum Neutrino Energy } \\
(\mathrm{MeV})\end{array}$ & $\begin{array}{c}\text { Predicted Total Flux } \\
\left(\mathrm{cm}^{-2} \mathrm{sec}^{-1}\right)\end{array}$ \\
\hline$p+p \rightarrow d+e^{+}+\nu_{e}$ & 0.42 & $6.1 \times 10^{10}$ \\
$p+e^{-}+p \rightarrow d \nu_{e}$ & 1.44 & $1.5 \times 10^{8}$ \\
$e^{-}+{ }^{7} \mathrm{Be} \rightarrow{ }^{7} \mathrm{Li}+\nu_{e}$ & 0.86 & $4.0 \times 10^{9}$ \\
${ }^{8} \mathrm{~B} \rightarrow{ }^{8} \mathrm{Be}+e^{+}+\nu_{e}$ & 14.10 & $5.6 \times 10^{6}$ \\
${ }^{13} \mathrm{~N} \rightarrow{ }^{13} \mathrm{C}+e^{+}+\nu_{e}$ & 1.15 & $5 \times 10^{8}$ \\
${ }^{15} \mathrm{O} \rightarrow{ }^{15} \mathrm{~N}+e^{+}+\nu_{e}$ & 1.73 & $4 \times 10^{8}$ \\
\hline
\end{tabular}

where $L_{v}$ is termed the vacuum oscillation length and is equal to

$L_{v} \equiv \frac{2 \pi 2 p}{\left|m_{2}^{2}-m_{1}^{2}\right|}=\frac{2.48}{\delta m^{2}} \frac{E_{\nu} \mathrm{MeV}}{\mathrm{eV}^{2}}$ meters

Thus, the sun allows investigation of $\delta m^{2} 10^{8}$ times smaller than can be investigated via terrestrial-based sources. However, due to our imperfect knowledge of the sun, one might properly infer from the above discussion that to observe neutrino oscillations from the sun, the mixing angle would have to be large, say $\sin ^{2} 2 \theta_{v}>0.1$. However, for small 


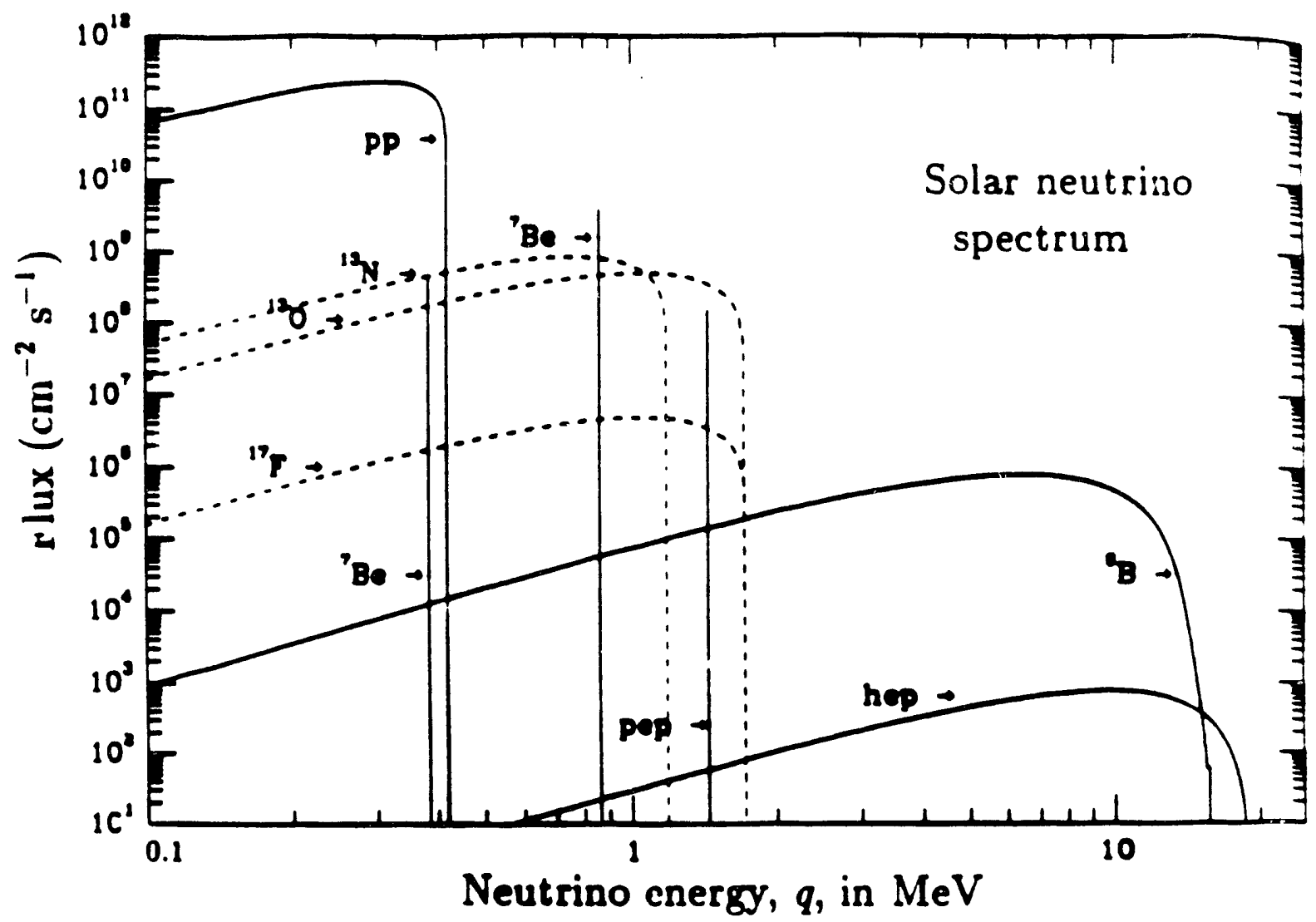

Figure 4. Plot of the solar neutrino flux at earth.

values of $\Delta m^{2} / 2 p$, the high density of matter (electrons) in the sun can greatly amplify the mixing via the so-called Mikhail-Smirnov-Wolfenstein [11] effect.

At the time of this talk, three solar neutrino detectors have taken data. They are listed in Table 4, with their neutrino energy thresholds and the period over which they have been accumulating data.

The physical characteristics of the ${ }^{37} \mathrm{Cl}$ detector are listed in Table 5. Standard solar model calculations of the neutrino flux, $\phi_{s}$, along with knowledge of the nuclear transition rates for ${ }^{37} \mathrm{Cl}+\nu_{e} \rightarrow{ }^{37} \mathrm{Ar}+e$ predict [9]

$\left\langle 0_{9} \sigma_{37} \mathrm{Cl}\right\rangle=7.9 \pm 3.3 \mathrm{SNU}$.

A SNU is a rate of $10^{-36}$ per sec per arget atom. The error in the expression above is estimated to be $3 \sigma$. Figure 5 is a graph of the measured ratio of the measured ${ }^{37} \mathrm{Ar}$ production rate over an 18-year period [9]. It should be noted that this is based on a total of 774 detected events, of which somewhat more than half (435) are background. The observed production rate of ${ }^{37} \mathrm{Ar}$ corresponds to $2.2 \pm 0.3(1 \sigma) \mathrm{SNU}$, well below the predicted rate, as is shown in Fig. 5. The ratio of the observed to predicted rate is

$\frac{\left\langle 0, \sigma^{37} \mathrm{Cl}\right\rangle_{\text {obs }}}{\left\langle 0, \sigma^{37} \mathrm{Cl}\right\rangle_{\mathrm{SSM}}}=0.28 \pm 0.05$ 
Table 4

Three ongoing solar neutrino experiments

\begin{tabular}{lll}
\hline & Threshold (MeV) & Status \\
\hline (1) $\nu_{e}+{ }^{3 i} \mathrm{Cl} \rightarrow e+{ }^{37} \mathrm{Ar}$ & $0.814(5.8)$ & $20 \mathrm{y}$ \\
$(2) \nu+e \rightarrow \nu^{\prime}+e$ & 7.5 & $4 \mathrm{y}$ \\
(3) $\nu_{e}+{ }^{i 1} \mathrm{Ga} \rightarrow e+{ }^{i 1} \mathrm{Ge}$ & 0.236 & 6 mo \\
\hline
\end{tabular}

Table 5

Characteristics of the ${ }^{37} \mathrm{Cl}$ detector

Location

Depth

Tank

Detector fluid

Total weight of fluid

Volume

Threshold

${ }^{37} \mathrm{Cl}$ atoms

Half-life ${ }^{37} \mathrm{Al}$

Neutrino sensitivity
Homestake Mine, Lead, South Dakota $4850 \mathrm{ft}$ $20 \mathrm{ft}$ diameter $\times 48 \mathrm{ft}$ long $\mathrm{C}_{2} \mathrm{Cl}_{4}$ 615 tons $3.8 \times 10^{5}$ liters $0.814 \mathrm{MeV}$ $2.16 \times 10^{30}$ 35.0 days ${ }^{8} \mathrm{~B},{ }^{7} \mathrm{Be}$

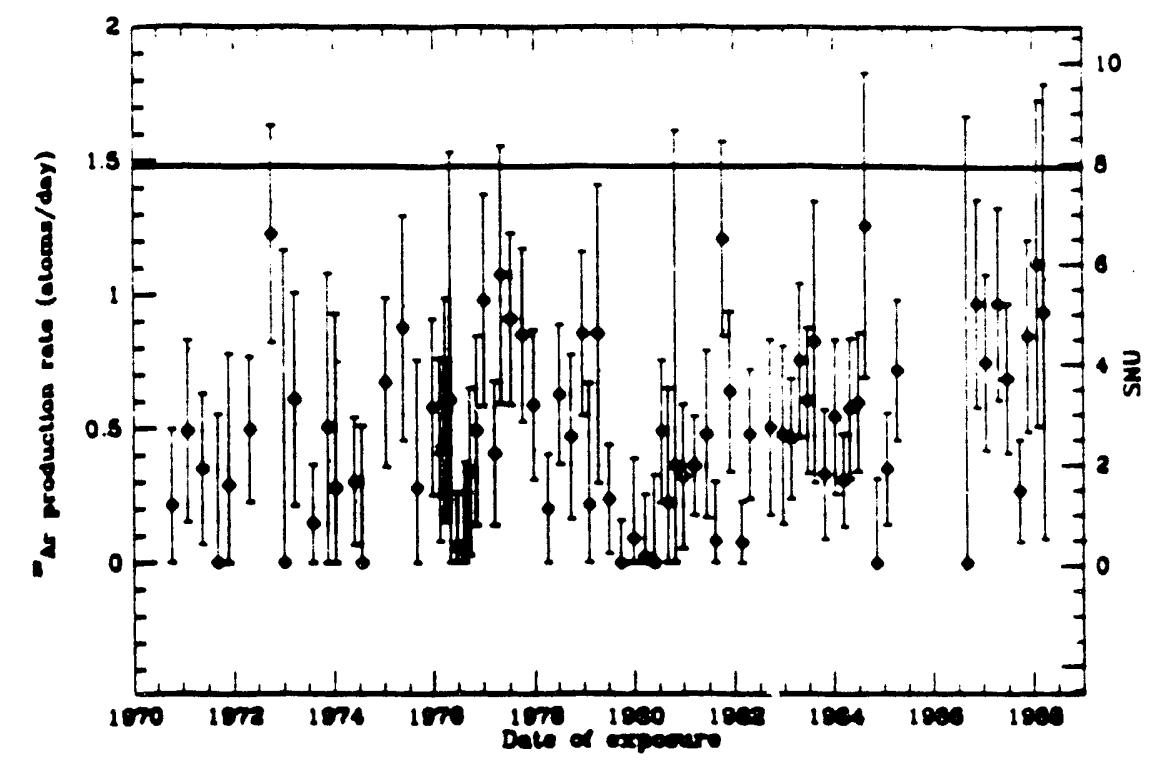

Figure 5. Diagram of the measure ${ }^{37} \mathrm{Ar}$ production rate in the ${ }^{37} \mathrm{Cl}$ experiment as a function of time. The solid line at $7.9 \mathrm{SNU}$ is the standard solar model prediction. 
where only a $1 \sigma$ error has been taken for the SSM prediction. The strongest transition between ${ }^{3 i} \mathrm{Cl}$ and ${ }^{3 i} \mathrm{Ar}$ occurs between the ${ }^{3 i} \mathrm{Cl}$ ground state and its analog states in ${ }^{37} \mathrm{Ar}$. These levels are separated by $5.8 \mathrm{MeV}$. So, although the threshold for a ${ }^{3 i} \mathrm{Cl}$ detector is $870 \mathrm{keV}$. it is most sensitive to neutrinos above $5.8 \mathrm{MeV}$. Keeping that in mind. we give the ratio for the SS.I predictor to the observed ${ }^{3 i} A$ r production rate for ${ }^{8} \mathrm{~B}$ neutrinos only.

$\frac{\left\langle\phi_{s} \sigma_{37} \mathrm{Cl}\right\rangle_{\text {obs }}}{\left\langle\phi_{s} \sigma_{37} \mathrm{Cl}\right\rangle_{\mathrm{SSM}}{ }^{8} \mathrm{~B} \text { only }}=0.36 \pm 0.06$

The characteristics of the Kamiokande II detector are listed ${ }^{9}$ in Table 6 . Though designed as a detector for proton decay, it has, by dint of ingenuity and high photocathode coverage (20\%), become an excellent solar neutrino detector. The neutrinos are detected via the Cerenkov radiation from the struck electron in the process

$\nu+e \rightarrow \nu+e$

where $E_{e}>7.5 \mathrm{MeV}$. The cross section for $\nu_{e}$ interaction with an electron is about six times larger than for $\nu_{\mu}$ or $\nu_{\tau}$ because $\nu_{e}$ can exchange $W^{ \pm}$in addition to $Z^{0}$ vector bosons. The struck electron retains a sense of the direction of the incident neutrino so that one can investigate if the neutrino signal comes from the direction of the sun. The energy of the recoil electron is also a function of incident neutrino energy. The Kamioka group has published [12] results from three years of observation. Figure $6(\mathrm{a})$ shows the direction of recoiling electrons with $E_{e}>9.3$ as a function of $\cos \theta$, where $\theta$ is the angle between the electron direction and the direction to the sun. Figure 6(b) shows the spectrum of electrons with $\cos \theta_{s}>0.8$, and one can see that it gratifyingly looks like what one would expect for the decay of ${ }^{8} \mathrm{~B}$, though suppressed in magnitude compared to what one expects in the SSM.

Table 6

Characteristics of the Kamiokande II detector

Location

Depth

Detector fluid

Fiducial mass

Electron threshold

Number of electrons

Veutrino sensitivity

Detectors
Kamioka (Japanese Alps)

$1 \mathrm{~km}(2700$ m.w.e.)

$\mathrm{H}_{2} \mathrm{O}$

680 tons

7.5 . MeV

$2.27 \times 10^{32}$

${ }^{8} \mathrm{~B}$

20-in. diameter PMT

Cerenkov light 

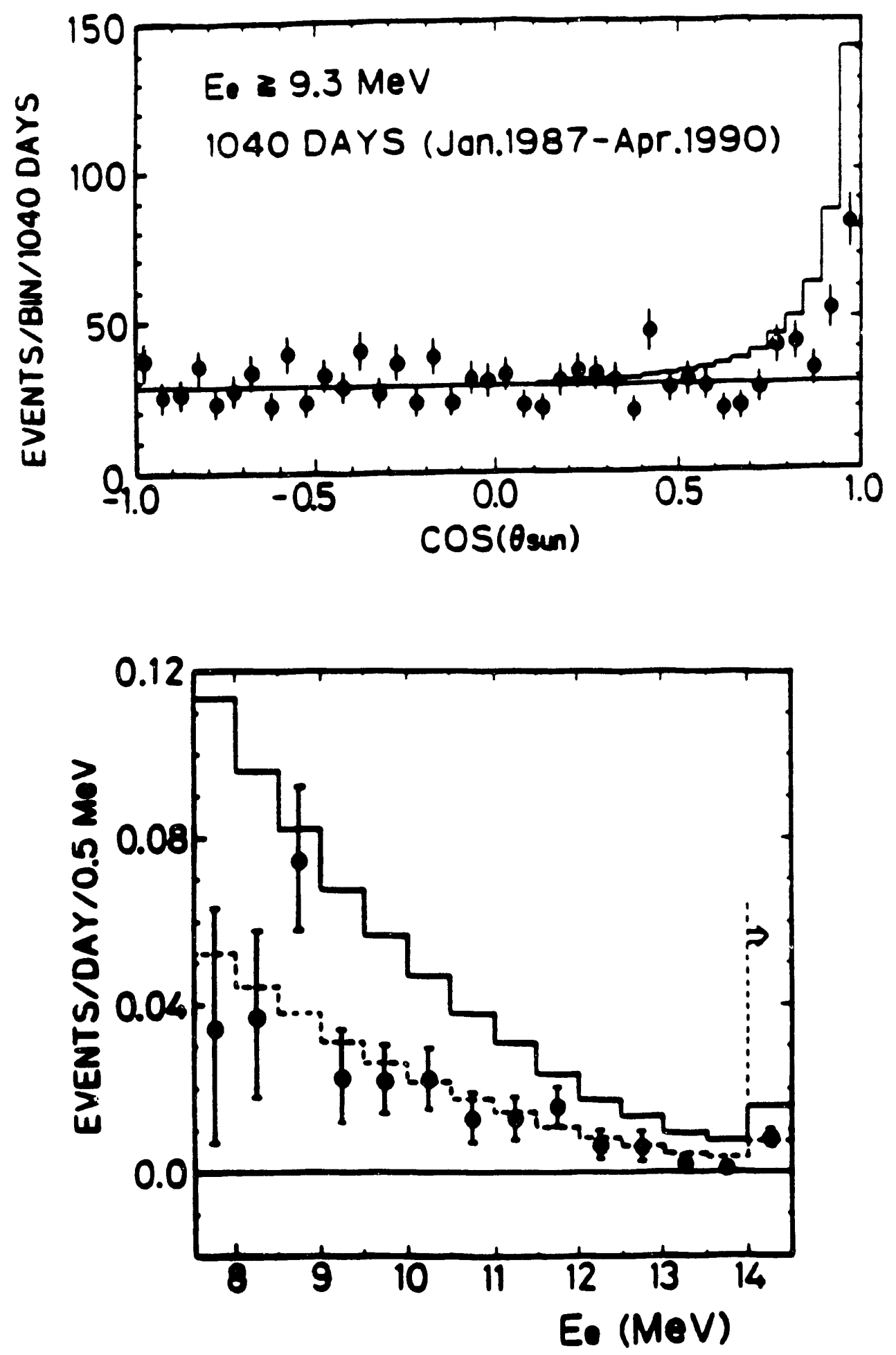

Figure 6. (a) The angular distribution of detected electrons above $93 \mathrm{MeV}$ with respect to the direction of the sun. (b) The energy distribution of electrons with large $\cos \theta$. The solid line is the prediction of the standard solar model, while the dashed histogram is that prediction reduced by 0.46 . 
Figure 7 shows Kamioka's data rate relative to the SSM prediction as a function of time. The rate is consistent with ieing constant in time and the ratio of observed to expected from the SSM calculation for ${ }^{8} \mathrm{~B}$ is

$\frac{\left\langle\phi \sigma_{\nu_{e}}\right\rangle_{\text {obs }}}{\left\langle\phi \sigma_{\nu_{e} t}\right\rangle_{\text {SSM }}}=0.46 \pm 0.05 \pm 0.06 \quad$ (Kamioka)

The errors are $1 \sigma$, the first statistical, the second systematic. Thus, the deficit observed in ${ }^{37} \mathrm{Cl}$ is confirmed and the two experiments can be readily made consistent with one another. One should bear in mind that the ${ }^{37} \mathrm{Cl}$ is only sensitive to $\nu_{e}$, while Kamiokande is sensitive to both $\nu_{e}$ and $\nu_{\mu}$. I will beg the question of the correlation of $\phi_{s}$ with the sunspot cycle, as it is by no means conclusive that there is any time variation.

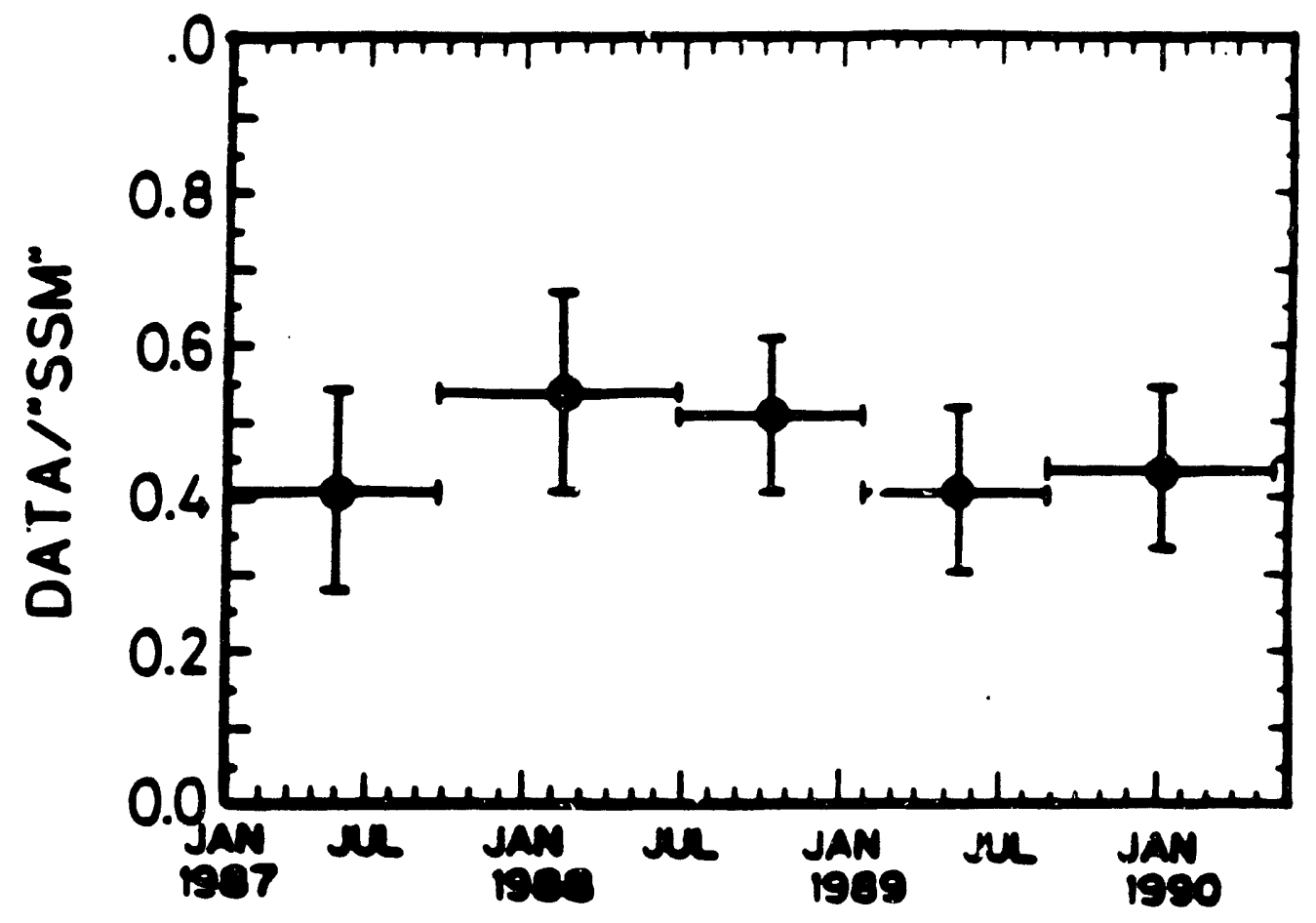

Figure 7. Ratio of the observed $\nu_{e}$ solar $\nu$ rate to that predicted ty the standard solar model as a function of time.

Taking these experiments at face value, the $\nu_{e}$ content of the solar flux from ${ }^{8} \mathrm{~B}$ decay is somewhere between $1 / 3$ and $1 / 2$ of the SSM prediction. A change of the central temperature of the sun by $3-4 \%$ would explain this, as would the more exotic explanation that the neutrinos are altered on their way out of the sun or on their way in transit to the earth. A way to examine this dilemma is to measure the flux of neutrinos from the solar $p+p \rightarrow d+e^{+}+\nu_{e}$ process. This flux depends on the sun's central 
temperature only as the 1.2 power: thus. one is certain of the number created to within a few percent by virtue of the observed ${ }^{8} \mathrm{~B} \nu_{e}$ fluxes. Any large reduction in this low energy flux must inrolve neutrino physics.

To observe the low-energy neutrino flux. gallium is employed as the detector material via the ${ }^{i 1} \mathrm{Ga}+\nu \rightarrow{ }^{i 1} \mathrm{Ge}+e$ reaction, which has a threshold of $233 \mathrm{keV}$. In this case. the ground state to ground state transition is both well known and strong. $\log f t=4.1$. so that the physical threshold is also a good representation of the detector sensitivity. Thus, gallium is fully capable of detecting the pp-generated neutrino flux. Two collaborations have been assembled to measure the solar neutrino flux using $\mathrm{Ga}$. One is called the Soviet-American Gallium Experiment [13] (SAGE) and the other is a largely European collaboration called GALLEX [14]. SAGE had an appreciable head start, as 60 tons of $\mathrm{Ga}$ were available in the Soviet Union to carry out the experiment. while GALLEX had to procure the gallium required for their project. The SAGE group has some six months worth of data on the solar neutrino flux. Fortunately for the purposes of this talk, the SAGE collaboration has produced a preprint [15] submitted for publication. which is used as the basis of reporting their results to this meeting.

Table $T$ lists the characteristics of the SAGE detector as it has operated in its initial stages. Table 8 lists the SSM prediction [9] for the rates expected in a Ga detector. Somewhat more than half the predicted yield comes from $p+p$ neutrinos. The reason that other processes contribute to ${ }^{i 1} \mathrm{Ge}$ production more strongly than their relative flux would suggest is the energy dependence of the production cross section.

$\sigma\left(\nu^{i 1} \mathrm{Ga} \rightarrow e^{71} \mathrm{Ge}\right)=$ const $\times p_{e}\left(E_{\nu}-0.233 \mathrm{MeV}\right)$

As natural $\mathrm{Ga}$ contains $39.9 \%{ }^{71} \mathrm{Ga}$, one expects $1.17^{71} \mathrm{Ga}$ atoms to be formed per day in 30 tons of $\mathrm{Ga}$ if the SSM flux is correct. In the SAGE experiment, the metallic $\mathrm{Ga}$ is in the liquid state and the ${ }^{71} \mathrm{Ge}$ is chemically extracted by adding a weak $\mathrm{HC}$ ] solution in the presence of hydrogen peroxide. This results in the extraction of $\mathrm{Ge}$ into the aqueous phase. This solution is then distilled, additional $\mathrm{HCl}$ is added, and an Ar purge is carried out, which sweeps the Ge out of the acid solution in the form of

Table $T$

Characteristics of the SAGE detector

Location

Depth

Detector fluid

Fluid mass

Threshold

${ }^{71} \mathrm{Ga}$ atoms

Half-life ${ }^{71} \mathrm{Ga}$
Mt. Andyrchi, North Caucasus, Russia 4700 m.w.e. Natural Ga $\left(39.9 \%^{71} \mathrm{Ga}\right)$

30 tons

$0.233 \mathrm{MeV}$ $10^{29}$

11.43 days 
Table 8

Capture rates predicted by the standard model for $\mathrm{a}^{71} \mathrm{Ga}$ detector

\begin{tabular}{cc}
\hline Neutrino Source & $\begin{array}{c}\text { Capture Rate } \\
\text { (SNU) }\end{array}$ \\
\hline$p p$ & 70.8 \\
$p e p$ & 3.0 \\
$h e p$ & 0.06 \\
${ }^{7} \mathrm{Be}$ & 34.3 \\
${ }^{8} \mathrm{~B}$ & 14.0 \\
${ }^{13} \mathrm{~N}$ & 3.8 \\
${ }^{15} \mathrm{O}$ & 6.1 \\
${ }^{17} \mathrm{~F}$ & 0.06 \\
\hline Total & $132 \mathrm{SNU}$ \\
\hline
\end{tabular}

$\mathrm{GeCl}_{4}$ and into 1.2 liters of $\mathrm{H}_{2} \mathrm{O}$. This is then converted to $\mathrm{GeH}_{4}$ (germane) from the extracted $\mathrm{GeCl}_{4}$. This gas sample is then mixed with xenon and injected into a lowbackground proportional counter with a volume of about $1 \mathrm{~cm}^{3}$. The efficiency of all the chemical processes is monitored by initially adding about $160 \mu \mathrm{g}$ of natural $\mathrm{Ge}$ to each of four reactors that contain the 30 tons of $\mathrm{Ga}$. The amount of carrier extracted can be measured both by atomic adsorption analysis and in the last stages by the volume of synthesized $\mathrm{GeH}_{4}$ that is produced. The overall extraction efficiency is typically $80 \%$ with an uncertainty of $\pm 6 \%$.

${ }^{71} \mathrm{Ge}$ decays via electron capture with a half life of 11.4 days. The capture is accompanied by emission of a $10.4-\mathrm{keV} K-x$ ray $40 \%$ of the time and by the emission of a $1.2-\mathrm{keV} L-x$ ray also $40 \%$ of the time. Unfortunately, the background in the region of the $L$ peak was too large for it to be used for determining the ${ }^{71} \mathrm{Ge}$ production rate. The typical background rate in the region of the $K$ peak is 0.1 events per day. Taking all efficiencies into account, the SSM would predict that SAGE should detect four ${ }^{71} \mathrm{Ge}$ in an extraction after the $\mathrm{Ga}$ has been exposed for, say, 30 days since it was last extracted. When the $\mathrm{Ga}$ was above ground, a relatively large amount of ${ }^{68} \mathrm{Ge}$ was produced via the hadronic component of cosmic radiation. ${ }^{68} \mathrm{Ge}$ has a half life of 271 days and also decays via electron capture, so its decay signal (apart from half-life) is identical with ${ }^{71} \mathrm{Ge}$; hence, it must be very thoroughly removed before ${ }^{71} \mathrm{Ge}$ production measurements can proceed. When initially brought underground, the rate of ${ }^{68} \mathrm{Ge}$ decay was more than $10^{4}$ higher than what is expected for the rate from solar neutrino production of ${ }^{71} \mathrm{Ge}$. The chemical extraction of ${ }^{68} \mathrm{Ge}$ began in the summer of 1988 and by January 1990 the experiment was ready to start. 
Figure 8 shows a time histogram of the integrated number of events in the ${ }^{i 1} \mathrm{Ge} \mathrm{K}$ peak windows from each of the extractions. There is no clear indication of a signal with a 11.4-day half life. which is perhaps not too surprising in that each run is expected to have only approximately four events due to ${ }^{i 1} \mathrm{Ge}$ decay. A statistical analysis of the data yields the statistical upper limit shown in Table 8. As a further check on the extraction

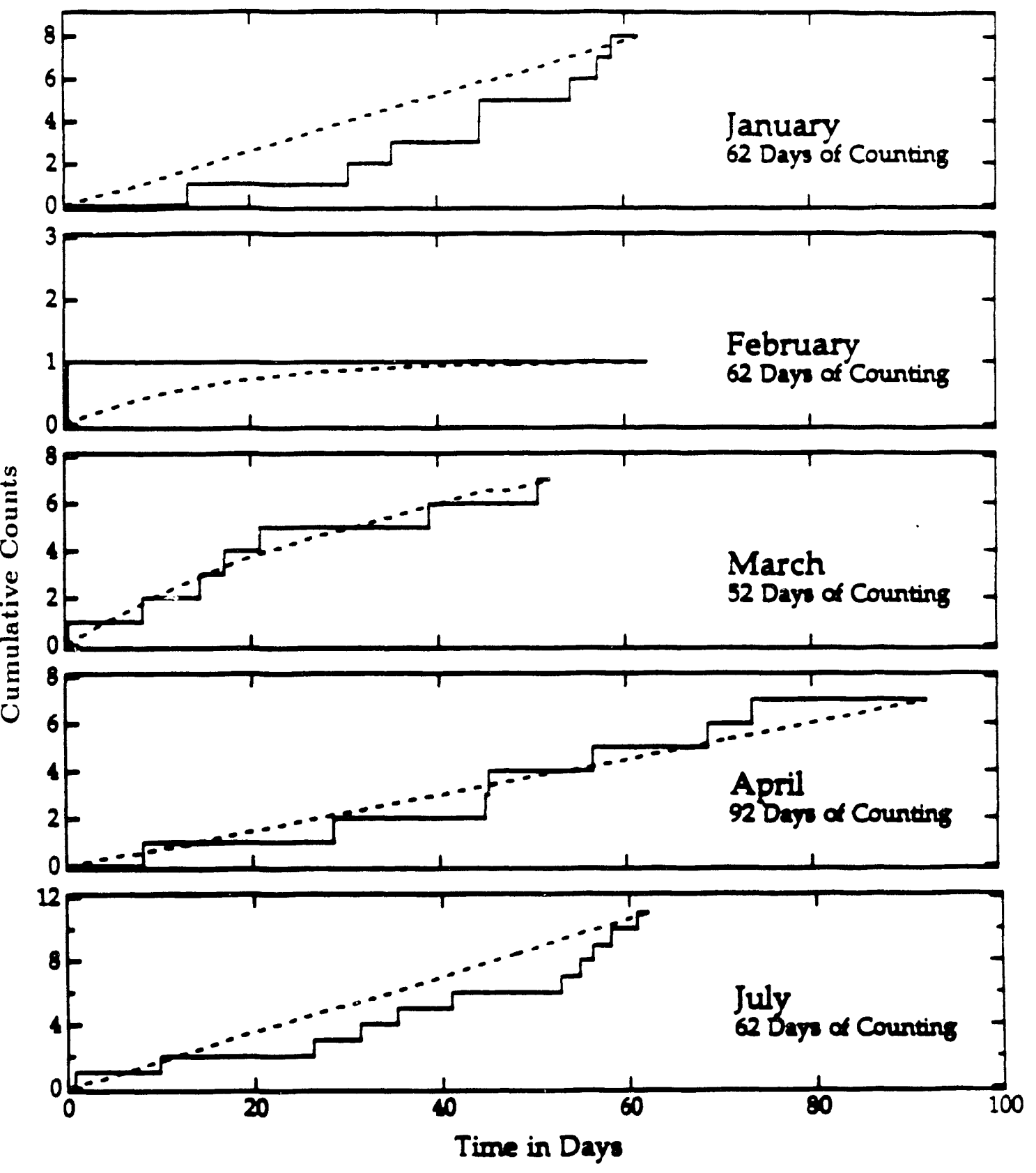

Figure 8. The integrated number of events satisfying the ${ }^{71} \mathrm{Ge}$ pulse height and rise time cuts for the $K x$-ray signal. The individual plots represent the observation for the month in 1990 for which the extraction was made. 
and counting efficiency, a known amount of ${ }^{i 1} \mathrm{Ge}$ was put into a $\mathrm{Ga}$ reactor, extracted. and counted. Table 9 shows the results from that test, and one sees very good agreement between the efficiency determined by chemical analysis of the carrier ${ }^{71} \mathrm{Ge}$ and the actual counting of the much smaller amount of radioactive ${ }^{i 1} \mathrm{Ge}$ introduced into the $\mathrm{Ga}$. In addition to statistical uncertainties, there are systematic uncertainties in the lengthy procedure involved in this experiment. The largest of the systematic uncertainties has to do with the time dependence of the background. The statistical analysis fits the data with two components-one has an 11.4-day half life and the other is the background rate taken to be constant in time. A radioactive decay chain such as, for example. Ra can introduce episodic background. Though none of the events in that particular decay are well suited to produce background in the region of the $\mathrm{Ge} K$ peak. In order to investigate this possibility, the time dependence of a wide body of data with energies and rise times outside the 10.4-keV $x$-ray window were investigated. All observations are consistent with a background that is constant in time. However, in order to be conservative about possible time variation in the background, it was fitted to a polynomial expression for the time dependence of the background. This analysis led to a maximal change in the limits set on the ${ }^{i 1} \mathrm{Ge}$ production rate of $30 \mathrm{SNU}$ ( $68 \%$ C.L.) and $35 \mathrm{SNU}$ (90\% C.L.).

Table 9

Extraction efficiency test-Ge carrier versus ${ }^{71} \mathrm{Ge}$ counting

\begin{tabular}{ccccc}
\hline & & \multicolumn{2}{c}{ Efficiency $(\%)$} \\
\cline { 3 - 4 } Extraction \# & Ge Carrier $(\mu \mathrm{g})$ & ${ }^{71} \mathrm{Ge}$ (atoms) & Carrier & ${ }^{71} \mathrm{Ge}$ \\
\hline 1 & $410 \pm 10$ & $5188 \pm 195$ & $78 \pm 4$ & $79 \pm 5$ \\
2 & $97 \pm 2$ & $1131 \pm 107$ & $84 \pm 20$ & $84 \pm 26$ \\
3 & $21 \pm 2$ & $<200$ & \\
$\Sigma$ & $528 \pm 10$ & $6519 \pm 222$ & $101 \pm 5$ & -8 \\
Amount added & $525 \pm 26$ & $6555 \pm 359$ & & \\
\hline$\left({ }^{71} \mathrm{Ge}\right)_{\text {meas }}=11.0 \pm 2.4$ days (11.4 days).
\end{tabular}

The result of combining the statistical and systematic errors leads to a value $\left\langle\phi_{9} \sigma_{71} \mathrm{Ga}\right\rangle=20+15 /-20 \pm 32 \mathrm{SNU}$.

In terms of limits on the solar $\nu$ flux, this corresponds to

$$
\begin{aligned}
\left\langle\phi_{s} \sigma_{11 \mathrm{Ga}}\right\rangle & <55 \mathrm{SNU} \quad 68 \% \text { C.L. } \\
& <79 \mathrm{SNU} \quad 90 \% \text { C.L. }
\end{aligned}
$$


Taken at face value, this result demonstrates that the $\nu$ flux from the sun must undergo modification. There is one possible experimental escape. It could be the case that ${ }^{i 1} \mathrm{Ge}$ formed via $(\nu, \epsilon)$ production undergoes some unknown "hot atom" chemistry and forms a complex that cannot be extracted as efficiently as the Ge carrier used to determine the extraction efficiency. The most effective way to test this is to irradiate a sizable $\mathrm{Ga}$ sample ( $\sim 10$ tons) with an intense ( $\sim 1$. Megacurie) ${ }^{51} \mathrm{Cr}$ source. ${ }^{51} \mathrm{Cr}$ decays by electron capture, emitting a $746-\mathrm{keV}$ neutrino $90 \%$ of the time and a $426-\mathrm{keV}^{-}$ neutrino the other $10 \%$. An engineering run was carried out at SAGE with a fraction of a Megacurie source in the fall of 1990 but produced insufficient data to permit a measurement of the extraction efficiency. A calibration run will be carried out in late 1992 or 1993.

The results of the three solar neutrino experiments just discussed can be accounted for by taking account of matter-induced neutrino oscillation in the sun. Boris Kayser will discuss that in his presentation. I want only to list the conclusion [16] of that analysis,

$\Delta m^{2} \sin ^{2} 2 \theta_{v}=4 \times 10^{-8 \pm 1} \mathrm{eV}$.

A not unreasonable and consistent interpretation of these results would be

$$
\begin{aligned}
m_{\mu} & \sim 10^{-3} \mathrm{eV} \\
m_{e} & \ll 10^{-3} \mathrm{eV} \\
\theta_{v} & \simeq 0.1
\end{aligned}
$$

Thus, these experiments show $m_{\nu} \neq 0$ and that lepton flavor is not conserved. If correct, this is the first observed breakdown of the minimal standard model and hence is an extremely important result.

The task of future research would then lie in measuring the neutrino masses and mixing angles in a manner similar to the K.M.C. mass matrix for the quark sector. However, the first order of business must be to generate more robust results from the $\mathrm{Ga}$ experiments. The SAGE experiment is now back in operation employing the full complement of 60 tons of $\mathrm{Ga}$ as a detector. The GALLEX experiment received final shipment on its 30 tons of $\mathrm{Ga}$ in March of 1990 and has reduced the ${ }^{68} \mathrm{Ge}$ background to a sufficiently low level that it is now sensitive to ${ }^{71} \mathrm{Ge}$ production via solar neutrinos. The counting systems in the GALLEX experiment have sufficiently low noise and low background that they can count the $L-x$ ray, as well as the $K-x$ ray. Hence, both experiments are of comparable sensitivity and within a year a good value for $\left\langle\phi_{,} \sigma_{71} \mathrm{Ga}_{\mathrm{a}}\right\rangle$ will be to hand. Then with a good calibration of each experiment, the issue should be settled. 


\section{THE 17-keV NEUTRINO}

The $17-\mathrm{keV}$ neutrino rides again! While the solar neutrino results can be incorporated into the MSM via a straightforward extension, the $17-\mathrm{keV}$ neutrino is a real outsider. It first appeared on the scene some six years ago when Simpson reported [1i] on an anomaly in the spectrum of ${ }^{3} \mathrm{H}$ decay. The ${ }^{3} \mathrm{H}$ was implanted in a $\mathrm{Si}(\mathrm{Li})$ detector. Subsequent experiments did not substantiate Simpson's claims of a 2-4\% admixed component of a $17-\mathrm{keV}$ neutrino into normal beta decays. Needless to say, a $17-\mathrm{keV}$ neutrino is a very difficult object to accommodate at this stage, as there are a variety of constraints on neutrino masses and mixing angles. Boris Kayser will discuss these constraints in his presentation, and I will deal with the experimental situation in the following.

One is compelled to point out what a tricky business beta decay experiments are. especially those that depend on measurement of the spectrum shape. Erroneous inferences have been drawn in the past regarding the electron antineutrino mass, the existence of second-class currents, and the present set of $\bar{\nu}_{e}$ mass measurements seems to be finding $m \frac{2}{\nu_{e}}<0$ as yet a further example of how hard it is to control systematic errors in these low-energy spectral measurements.

A paper [18] by Hime and Jelley earlier this year contains what appears to be very convincing data for a $17.0 \pm 0.4-\mathrm{keV}$ neutrino with a mixing angle of $\sin ^{2} \theta=0.0084 \pm$ $0.0006 \pm 0.0005$. The measurement was carried out on the decay of ${ }^{35} \mathrm{~S}\left(E_{0}=167 \mathrm{keV}\right)$, which is a better choice than ${ }^{3} \mathrm{H}$ as a source of $17-\mathrm{keV}$ neutrinos, as ${ }^{3} \mathrm{H}$ decay forces one to study electrons below $2 \mathrm{keV}$. In [17], the source was external to the detector, which was again a $\mathrm{Si}(\mathrm{Li})$ detector. The detector had a $200-\mathrm{mm}^{2}$ active area and a thickness of $5 \mathrm{~mm}$. Very considerable attention was paid to chamber vacuum, beta collimation, source preparation, detector response, pile-up, etc. Figure 9 shows the results of spectra obtained in two different runs and a composite of the two runs relative to data less than $17 \mathrm{keV}$ below the endpoint. To understand these figures, first let us consider Fig. 10, which shows the local effect of a heavy neutrino on the beta spectrum. It has all the properties evidenced by Eq. (4) and is added with a weight of $\sin ^{2} \theta$ on top of the normal beta spectrum with $m_{\nu_{e}} \cong 0$. Thus, a best fit to the overall spectrum produces a local notch at the heavy neutrino threshold and then undershoots the spectrum where $\left(E_{0}-E_{e}\right)>\left(E_{0}-m_{\nu}\right)$. The shape of the notch is nontrivial and is a function of neutrino mass. Figures 9(a) and 9(b) show the ratio of the measured spectrum to the best fit obtained, assuming no heavy neutrino emission. There is a local notch some $17 \mathrm{keV}$ below the endpoint and data between this notch and the beta endpoint are well above the best fit.

Similar effects have been seen by an LBL, group who measured the spectrum of ${ }^{14} \mathrm{C}$ dissolved into a $\mathrm{Ge}(\mathrm{Li})$ crystal that is used as the detector and source. Their statistics are not as impressive as those of [18], but they also obtain [19] a notch at $17.1 \mathrm{keV}$ with a value of $\sin ^{2} \theta \sim 1.3 \%$. 


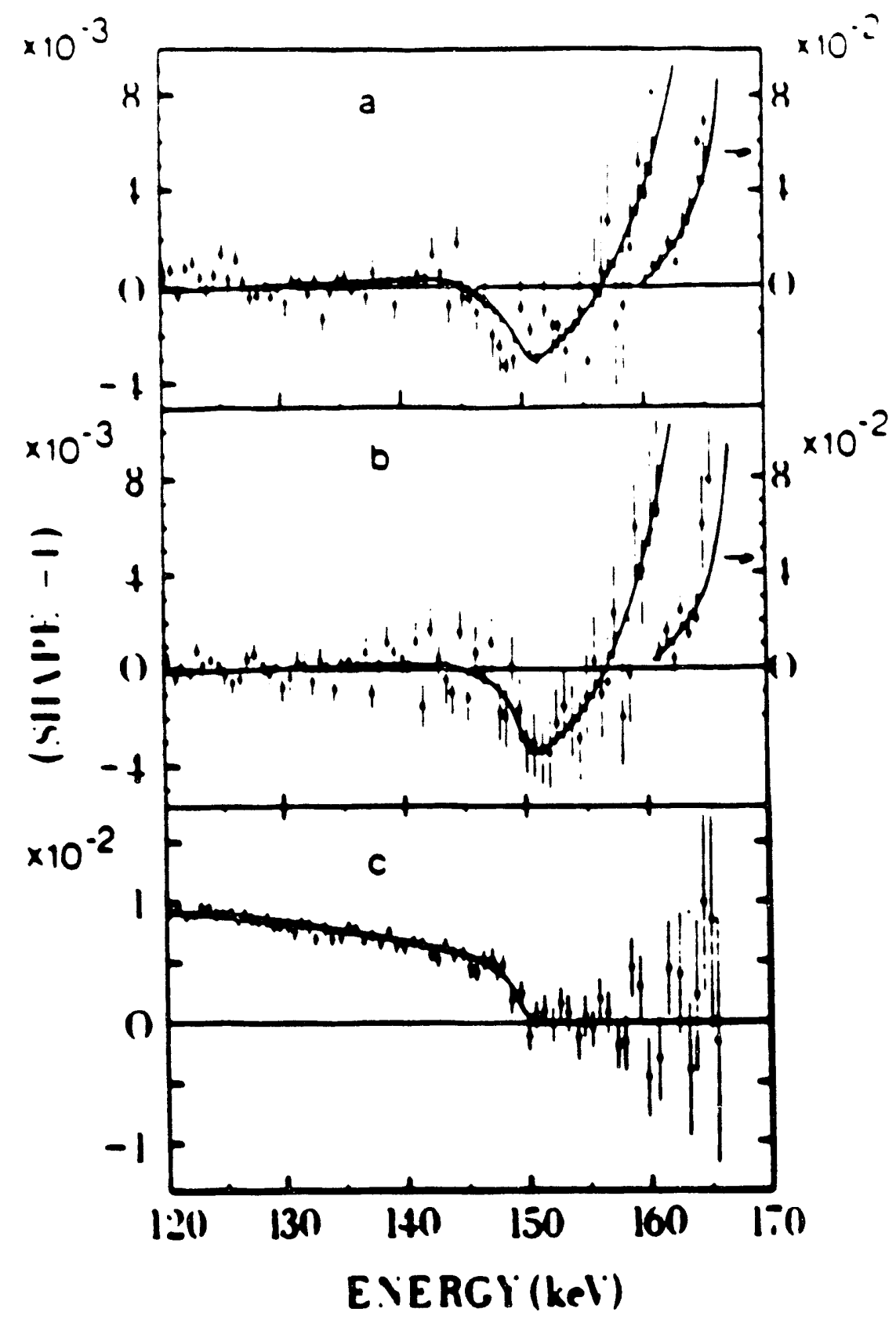

Figure 9. (a) Ratio minus one of the observed spectra to a calculated spectrum assuming that $\sin ^{2} \theta=0$ (Run 1). (b) Ratio minus one of the observed spectra to a calculated spectrum assuming that $\sin ^{2} \theta=0$ (Run 2). (c) Ratio minus one of observed spectra fitted to a calculated spectrum using the data at $E_{e}>150 \mathrm{keV}$ to determine the fit. The solid line through the data points is for $\sin ^{2} \theta=0.009$ (Runs 1 and 2). 

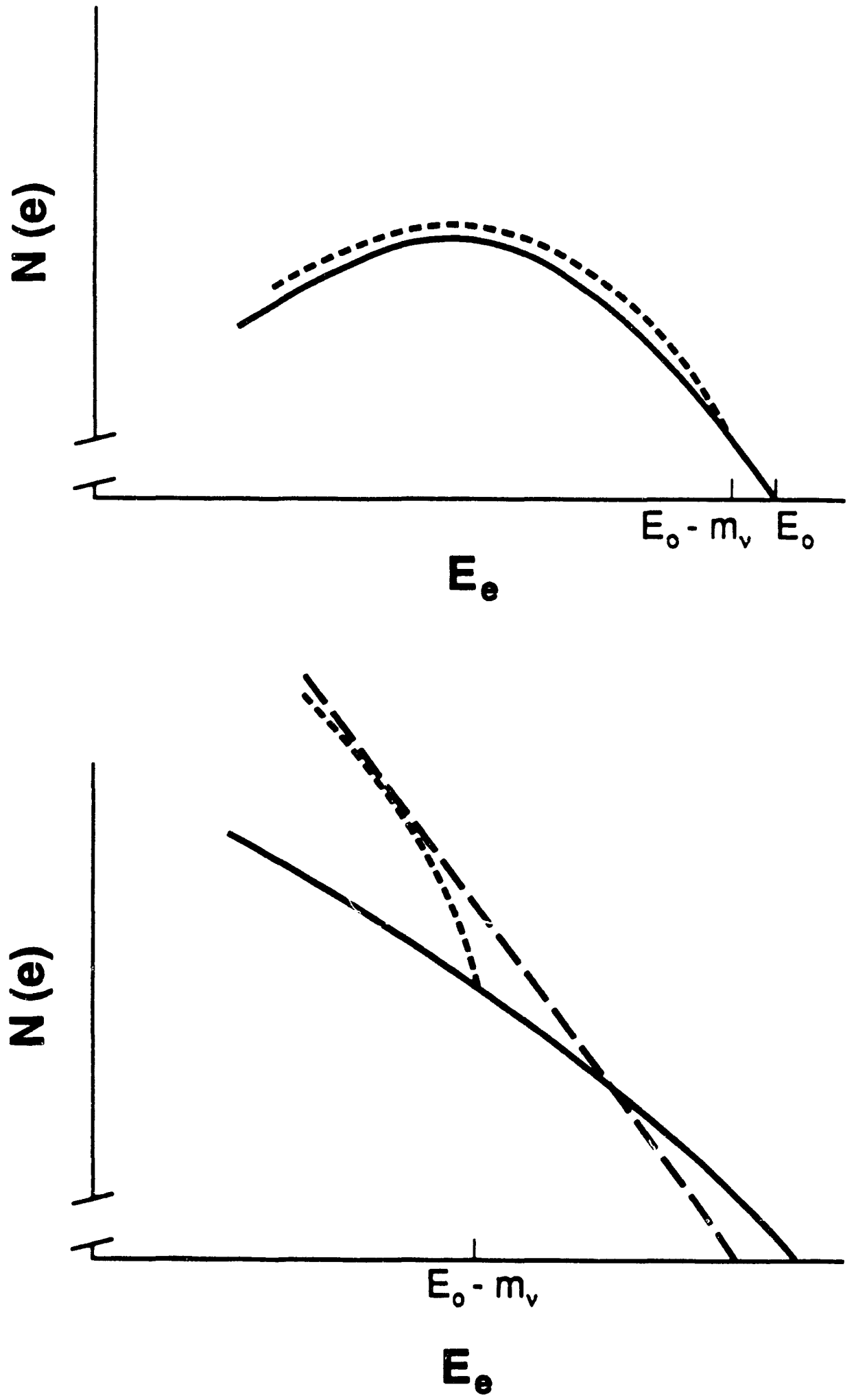

Figure 10. Schematic figure showing the effects of a heavy neutrino on the beta spectrum. 
In a recent publication. a Yugoslavian group reported [20] observation of a spectral modification of the inner bremsstrahlung following electron capture. Again, the statistics are not as large as those in [18]. but the analysis yields a $18-\mathrm{keV}$ neutrino with a mixing angle $\sin ^{2} \theta=0.0160 \pm 0.0053(1 \sigma)$. In this case, they measured the internal bremsstrahlung following capture in ${ }^{i 1} \mathrm{Ge}$ (endpoint $\sim 230 \mathrm{keV}$ ). The photons were detected in a 4.7 -cm-diameter, 3.65 -cm-thick Ge detector.

All of the experiments mentioned above are difficult; the significant data are within $30 \mathrm{keV}$ of the endpoint where the signal is usually small. There is, in each of their fits. some small linear or quadratic correction term to account for imperfect detector response. There is no apparent reason why or how these corrections would introduce anomalies into the spectrum of the kind reported. The one common feature of all these experiments is that the spectra have been measured with semiconducting crystalline detectors. Again, no known mechanism can explain the occurrence of a common anomaly in these three experiments. However, it should be borne in mind that no experiment using a magnetic spectrometer has observed any positive evidence for a heavy neutrino.

The most recent data from a magnetic spectrometer is contained in a preprint [21] from the Caltech group, who have examined ${ }^{35} \mathrm{~S} \rightarrow{ }^{35} \mathrm{Cl}+e+\nu$, the same decay as Hime and Jelley. They use a $\pi \sqrt{2}$ iron-free double focusing spectrometer to measure the ${ }^{35} \mathrm{~S}$ beta spectrum. Their setup has a momentum resolution of $0.27 \%$ (FWH.M), or an energy resolution of approximately $0.9 \mathrm{keV}$ at the threshold energy for $17-\mathrm{keV}$ neutrino emission. The data have high statistics, approximately $10^{6}$ counts per every $1.4 \mathrm{keV}$ in the $17-\mathrm{keV}$ neutrino threshold region. The measured spectrum is fit using both a linear $\left(K_{1}\right)$ and a quadratic $\left(K_{2}\right)$ function of $p_{e}$ to account for smooth variation in the spectrometer response, as well as different admixtures of a $17-\mathrm{keV}$ neutrino. These small detector response constants change value depending on what is assumed for the magnitude of the mixing angle for the $17-\mathrm{keV}$ neutrino. Thus, for $\sin ^{2} \theta=0$, $K_{1}=9.9 \times 10^{-4} \mathrm{keV} / c$ and $K_{2}=-3.8 \times 10^{-6}(\mathrm{keV} / \mathrm{c})^{2}$, while for $\sin ^{2} \theta=0.0085$, $K_{1}=6.3 \times 10^{-3}$ and $K_{2}=-1.7 \times 10^{-6}$. The $\chi^{2}$ for the fits are not impressive in either case $-\chi^{2}=303$ for $\sin ^{2} \theta=0$ and 458 for $\sin ^{2} \theta=0.0085$ for 81 degrees of freedom for both cases. The $\chi^{2}$ fit would appear to strongly favor $\sin ^{2} \theta=0$ relative to $\sin ^{2} \theta=0.0085$, but the overall large value for $\chi^{2}$ is disturbing. Figure 11 shows their data and the fit expected for a $17-\mathrm{keV}$ neutrino present with a mixing angle of 0.0085 . They claim that at $90 \%$ C.I., $\sin ^{2} \theta \leq 0.0060$, well below the value claim 2 d by [17].

For me, the most compelling evidence against the need for a heavy neutrino component in nuclear beta decay is the earlier work [22] of a Chalk River group. They also employed a $\pi \sqrt{2}$ iron-free beta spectrometer, shown in Fig. 12, to study the decay of ${ }^{63} \mathrm{Ni}\left(E_{0}=67 \mathrm{keV}\right)$. The energy resolution is the order of $100 \mathrm{eV}$ and 900 data points were taken between 25 and $70 \mathrm{keV}(60 \mathrm{eV} / \mathrm{step}$ at $50 \mathrm{keV})$. Some $10^{8}$ total events were measured. The Kurie plots of the data are shown in Fig. 13. Figure 14 shows the fits to the data from their article, and it is obvious that a mixing as large as the value being claimed at that time $\left(\sin ^{2} \theta=0.03\right)$ is incompatible with the data. Figure 15 shows a best fit value for $\sin ^{2} \theta$ as a function of the mass of a possible heavy neutrino. At 


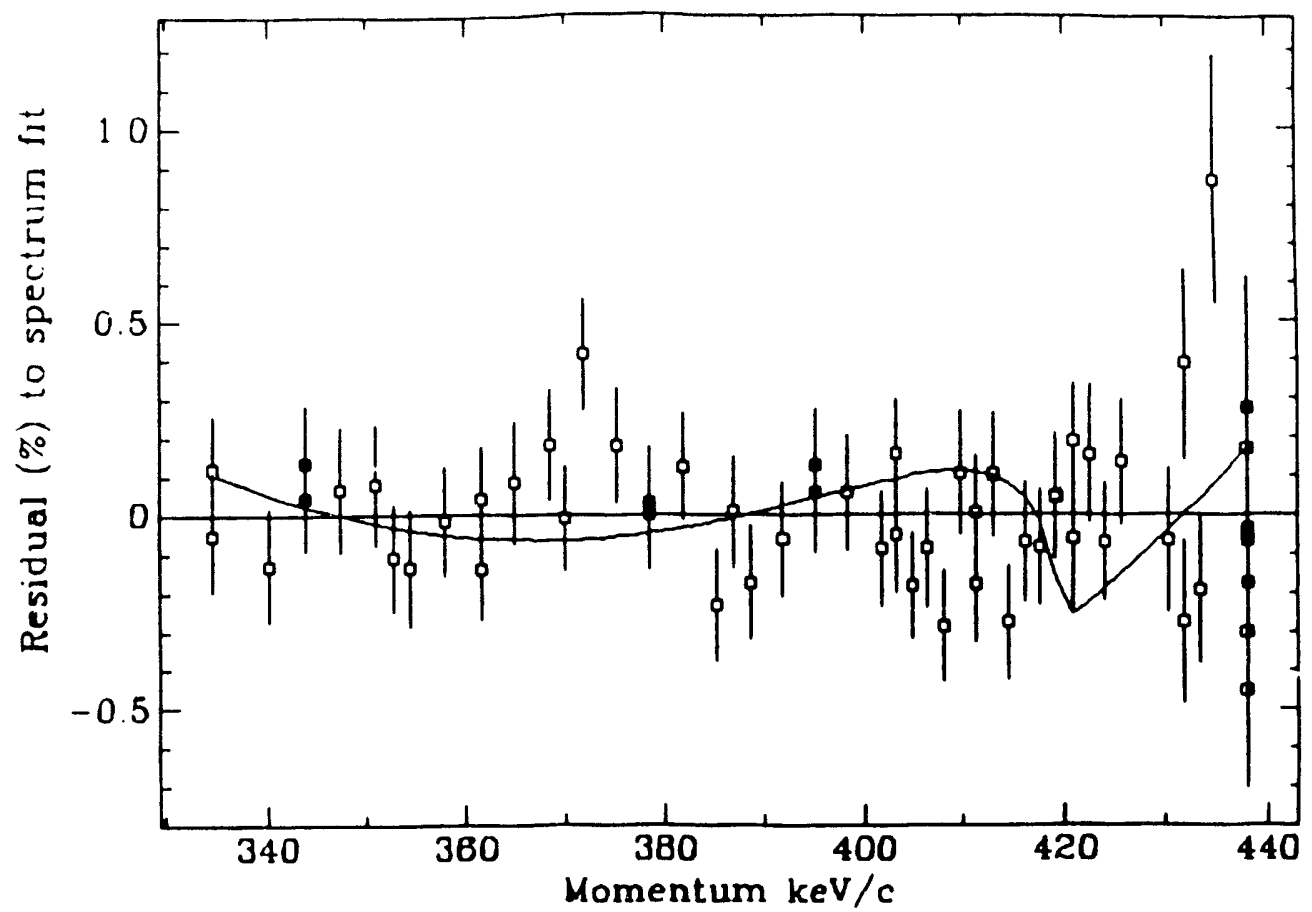

Figure 11. Residuals in percent from the observed spectra of beta particles from ${ }^{35} \mathrm{~S}$, assuming no heavy neutrino components. The curve represents how the deviation would occur for a $17-\mathrm{keV}$ neutrino with $\sin ^{2} \theta=0.0085$.

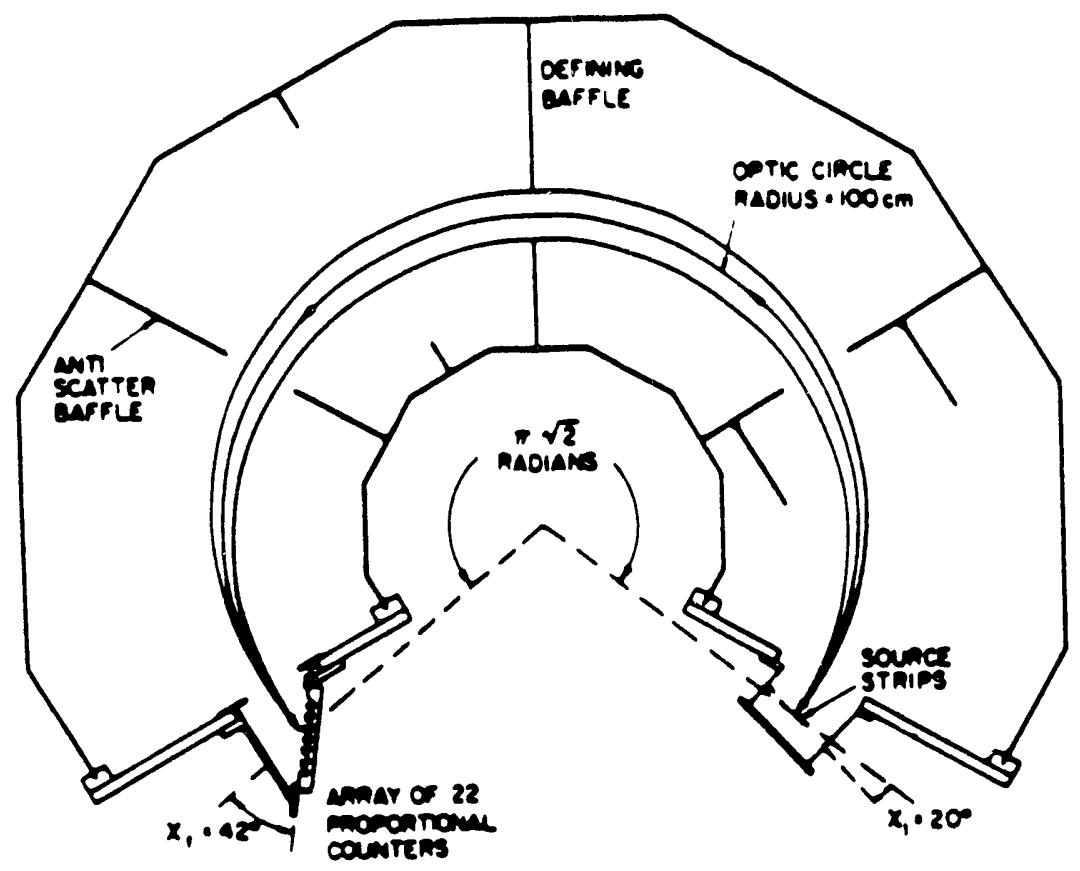

Figure 12. Plan section of the Chalk River iron-free $\pi \sqrt{2}$ beta spectrometer. 

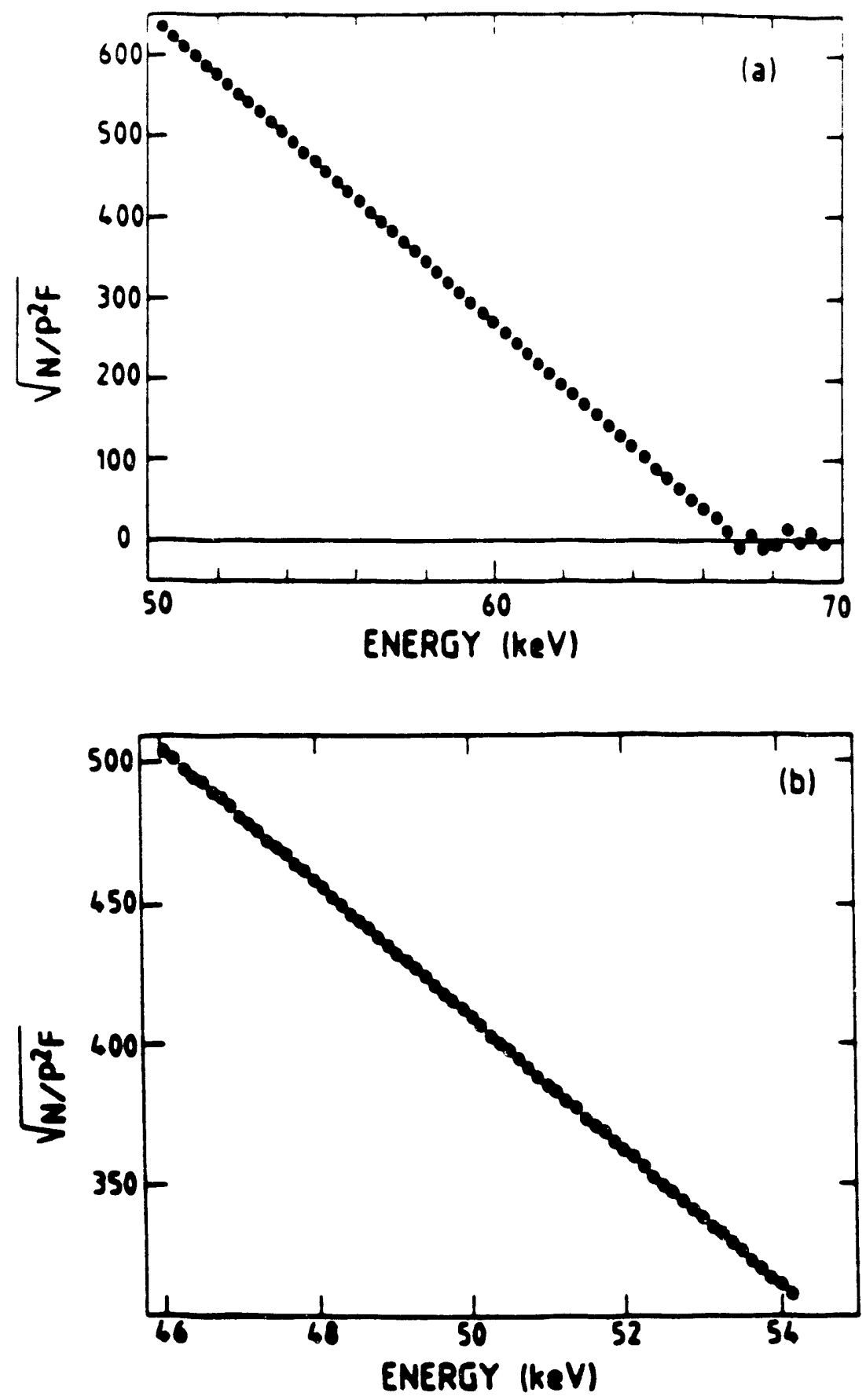

Figure 13. (a) Kurie plot of the measurements for ${ }^{63} \mathrm{Ni}$ beta decay. (b) Detail of the Kurie plot in the region of $E_{\beta}=50 \mathrm{keV}$, where the effects of a $17-\mathrm{keV}$ neutrino would be expected to be manifest. 

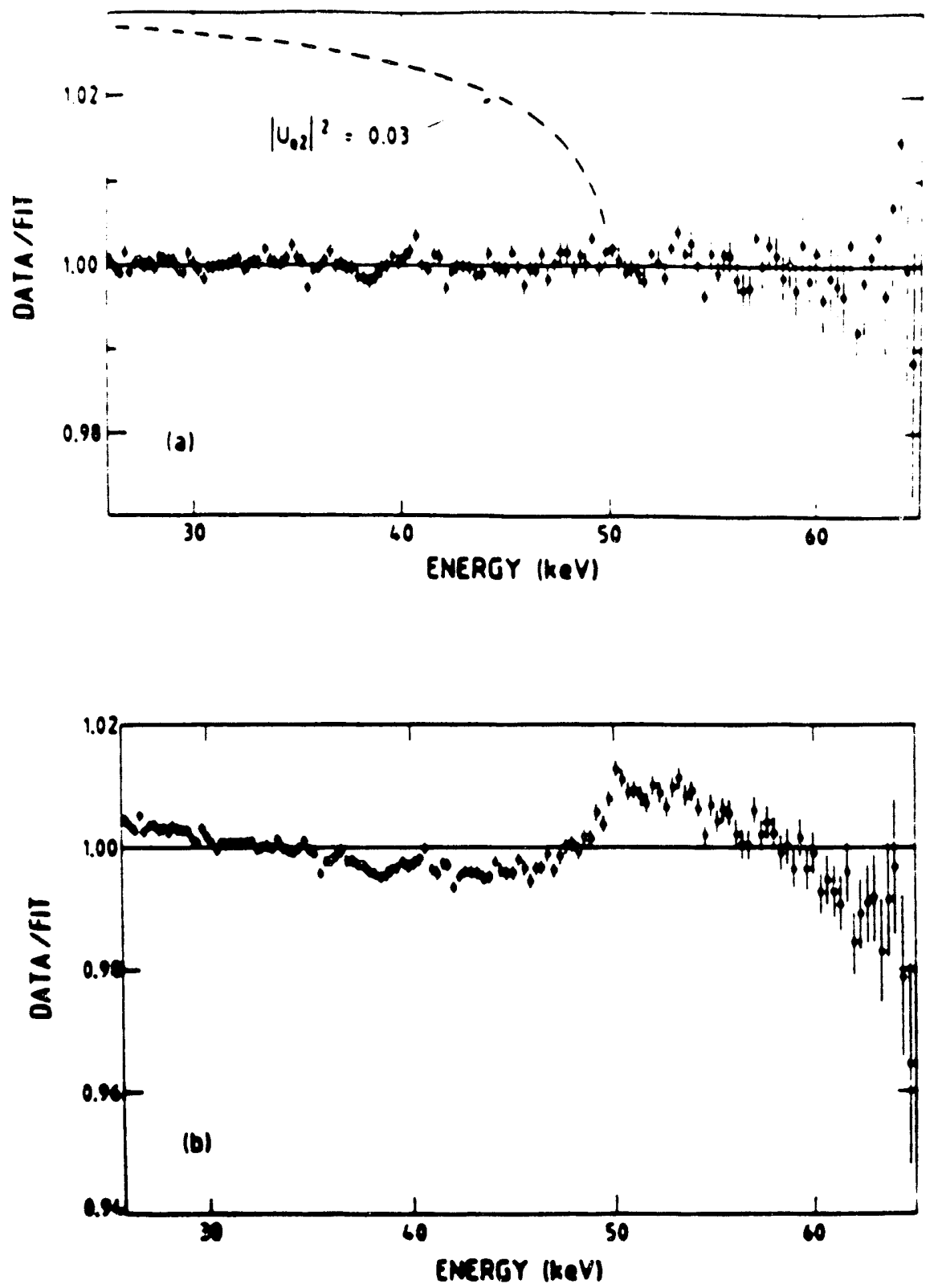

Figure 14. (a) Ratio of the observed data to a calculated spectrum, assuming no heavy neutrino $\left(\sin ^{2} \theta=0\right)$. (b) Ratio of the observed data to a calculated spectrum, assuming a $17-\mathrm{keV}$ neutrino with $\sin ^{2} \theta=0.03$. 


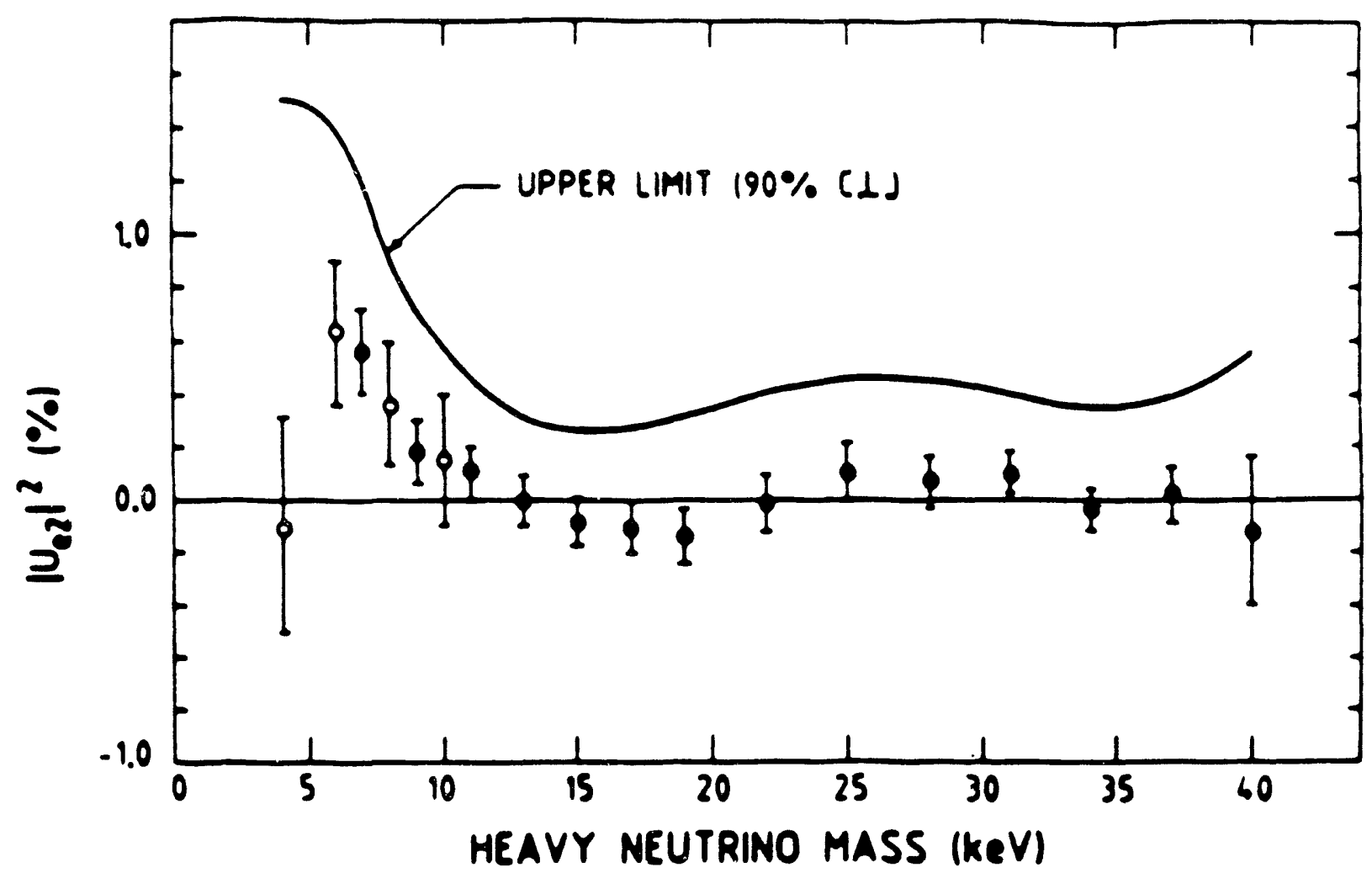

Figure 15. Residuals of fits to the observed ${ }^{63} \mathrm{Ni}$ beta decay spectrum, assuming different values of heavy neutrino masses and mixing angles. The solid line shows the $90 \%$ confidence level on the upper limit of the possible $\sin ^{2} \theta$ for a value of the heavy neutrino mass.

$17 \mathrm{keV}$, the $90 \%$ confidence limit on $\sin ^{2} \theta$ would appear to be $0.3 \%$, again well below the value claimed in [18].

There have been other measurements reporting limits on a $17-\mathrm{keV}$ component to nuclear beta decay. It can be saic that every report of a positive effect occurs when a crystalline semiconducting detector has been used. No magnetic spectrometer measurement has confirmed the existence of a heavy-neutrino component. It is also fair to say, even though the subject has been given a great deal of thought, that no one has come forth with a definite idea of what mechanism in $\mathrm{Si}$ and Ge detectors can simulate the effect of a $17-\mathrm{keV}$ neutrino.

This is an exciting and interesting issue which, if true, would upset the standard model in a rather serious way. As you will learn from Boris Kayser, this $17-\mathrm{keV}$ neutrino does not easily fit into our prejudices or what we already know about neutrino properties. 


\section{5. $\nu p$ ELASTIC SCATTERING}

As this conference is entitled "Hadronic Structure and Electroweak Interactions." I think it appropriate to point out the use of the neutrino to reveal a very important aspect of the structure of the nicleon. Neutrino elastic scattering can be used to obtain the strange quark coniribution to the proton spin or more generally the $\mathrm{SU}(3)_{F}$ singlet axial vector form factor.

The report [23] of the EMC group of the violation of the Ellis-Jaffe [24] sum rule and the immediate corollaries of a sizable strange quark contribution

$2\left\langle S_{Z}\right\rangle_{s} \equiv \Delta s=\int_{0}^{1}[\vec{s}(x)+\overrightarrow{\bar{s}}(x)-\overleftarrow{s}(x)-\overleftarrow{s}(x)] d x=0.190 \pm 0.032 \pm 0.046$

to the proton spin and smill value for the sum of all quark spins in the proton

$\sum \Delta q=\Delta u+\Delta d+\Delta s=+0.12 \pm 0.094 \pm 0 .(13) 8$

has spurred great theoretical and experimental interest in the issue of how the nucleon spin is realized. 'The experiment reported by the EMC group is very difficult. It consists of measuring the asymmetry $(A)$ in the yield of longitudinally polarized muons from longitudinally polarized protons.

$A(x)=\frac{d \sigma(x) \uparrow i-d \sigma(x) \uparrow \uparrow}{d \sigma(x) \uparrow \downarrow d \sigma(x) \uparrow \uparrow}$

where $x$ is the fraction of momentum of the proton carried by the struck quark in the infinite momentum frame. The proton spin structure furction $g_{1}(x)$ is measured by

$g_{1}(x) \simeq A(x) F_{1}(x)$

Kaplan and Marohar [25] hare jointed out that neutrino clastic scattering can determine the strange quark cont ibution to the proton axial vector form factor. Actually, a very similar point had $\mathrm{k}$ sen made much earlier [26], but the context was lacking to motivate any meanicgful experiments. Neutrino-proton elastic scattering has been measured [25] but at a $Q^{2}$ that is too high, so that a variety of uncertainties enter the problem and render impossible interpreting the result in terms of a strange quark axial vector form factor. The expressions developed below follow a scheme similar to that used by Beise and McKeown [27].

In terms of quark currents, the neutral weak current (NWC) of a nucleon may be written as

$j_{. N}^{Z_{0}} \simeq\left\langle N^{\prime}\left|\sum_{i=r, d, s} \bar{q}_{i} \gamma^{\mu}\left(1-\gamma_{5}\right) t_{3 i} q_{i}-2 \sin ^{2} \theta_{W} Q_{i} \bar{q}_{i} \gamma^{\mu} q_{i}\right| N\right\rangle$, 
where $Q_{i}$ is the quark charge and $t_{3}$ is the weak : $\operatorname{sospin}\left(t_{3}(u)=\frac{1}{2} u, t_{3}(d)=-\frac{1}{2} d\right.$. $\left.t_{3}(s)=-\frac{1}{2} s\right)$. The first product in the sum is pure $V-A$, while the second is just $-2 \sin ^{2} \theta_{W}$ times the electromagnetic current. Thus, we can readily write the NWC in terms of known form factors and unknown strange quark contributions

$$
\begin{gathered}
j_{. V}^{Z_{0}} \simeq\left\langle V^{\prime}\right| \frac{-G_{A}\left(Q^{2}\right)}{2} \gamma^{\mu} \gamma^{5} \tau^{3}+\frac{\bar{s} \gamma_{\mu} \gamma_{5} s}{2}+\left(1-2 \sin ^{2} \theta_{W}\right) F_{v}^{\mathrm{EM}}\left(Q^{2}\right) \tau_{3} \\
+\left(-2 \sin ^{2} \theta_{W}\right) F_{s}^{\mathrm{EM}}\left(Q^{2}\right)-\frac{\bar{s} \gamma^{\mu} s}{2}|N\rangle .
\end{gathered}
$$

where $F_{v}^{\mathrm{EM}}\left(q^{2}\right)$ and $F_{s}^{\mathrm{EM}}\left(q^{2}\right)$ are the isovector and isoscalar electromagnetic form factors, respectively-a $\tau_{3}=+1$ for proton, -1 for neutron. Hence, $F_{v}^{\mathrm{EM}}\left(q^{2}\right)=$ $F_{1, v}\left(q^{2}\right) \nu^{\mu}+F_{2, v}\left(q^{2}\right)\left(\sigma_{\mu \nu} q_{\nu} / 2 m_{p}\right)$ and so forth. $G_{A}\left(Q^{2}\right)$ is the axial vector form factor of the nucleon and $\left(\bar{s} \gamma \gamma_{5} s / 2\right)$ and $\left(\bar{s} \gamma_{\mu} s / 2\right)$ a, e strange quark contributions to the axial vector and vector form factors, respectively. The sírange quark contributions are isoscalar.

In order to measure, say $\left(G_{s} / 2\right)$, the strange quark contribution to the axial current, it is essential to carry out the measurement of the yield of $\nu+p \rightarrow \nu+p$ at low $Q^{2}$, i.e., $\left(Q^{2}<0.1 \mathrm{GeV}^{2}\right)$. This minimizes the following uncertainties in the determining contributicns of $G_{s}\left(q^{2}\right)$, the uncertainty in $G_{A}\left(q^{2}\right)$ because of the uncertainty in $m_{A}=$ $1.03 \pm 0.04 \mathrm{GeV}$, and $F_{1}^{s}\left(q^{2}\right)$ and $F_{2}^{s}\left(q^{2}\right)$ are completely unknown. At $|Q| \ll m_{p}$, magnetic contributions are suppressed so that, using Eq. (17),

$\sigma(\nu p) \sim\left|\left\langle p^{\prime}\left|\left(\frac{-1.26}{2}+\frac{G_{s}}{2}\right) \gamma^{\mu} \gamma_{5}+\frac{1-4 \sin ^{2} \theta_{W}}{2} \gamma^{\mu}\right| p\right\rangle\right|^{2}$

Fortuitously, the charge form factor is seen to be much suppressed (0.04). In this representation, the value of $G_{g}(0)$ inferred from the EMC values [22] of $g_{1}(x)$ is

$G_{s}=0.19 \pm 0.08$

In a LAMPF experiment (E1173), $G_{s}(0)$ is expected to be measured to an accuracy of 0.05 via low-energy $\left(\nu_{\mu}=200 \mathrm{MeV}\right)$ neutrino-proton scattering. Notice that there was no need to use $\mathrm{SU}_{3}$ symmetry arguments in the case of $\nu p$ scattering. The strange quark contribution appears as an isoscalar contribution to the nucleon axial weak current.

\section{CONCLUSIONS}

I hope it has been apparent that neutrino physics, mostly at low energy, has proven to be interesting and revealing of very fundamental issues in the standard model. Further, there are more potentially interesting questions presently being addressed than is the case for most areas of nuclear and particle physics. 
In addition to their own intrinsic properties, neutrinos allow investigation of new and unique properties of the structure of the nucleon.

\section{REFERENCES}

1 F. Dydak, these proceedings.

2 V. A. Lubimov et al., Phys. Lett. B94 (1980) 266.

3 S. Boris et al., Phys. Rev. Lett. 58 (1987) 2019.

4 H. Kawakami et al., Phys. Lett. B256 (1991) 105.

5 R. G. H. Robertson et al., Phys. Rev. Lett. 67 (1991) 957.

6 R. L. Martin and J. S. Cohen, Phys. Lett. A110 (1985) 95.

7 O. Fackler et al., Phys. Rev. Lett. 55 (1985) 1388.

8 H. Agren and V. Canavetta, Phys. Rev. A38 (1988) 2707.

9 J. N. Bahcall, Neutrino Astrophysics, Cambridge University Press, 1989.

10 B. Pontecorov, Zh. Eksp. Teor. Fiz. 53 (1967) 1717 [Sov. Phys. JETP 22 (1966) 1051].

11 L. Wolfenstein, Phys. Rev. D17 (1978) 2369; S. P. Mikheyev and A. Yu. Smirnov, Yad. Phys. 42 (1985) 1441 [Sov. J. Nucl. Phys. 44 (1986) 938].

12 K. S. Hirata et al., Phys. Rev. Lett. 65 (1990) 1297.

13 The SAGE Collaboration: A. I. Abazov, D. N. Abdurashitov, O. L. Anosov, L. A. Eroshkina, E. L. Faizov, V. N. Gavrin, A. V. Kalikhov, T. V. Knodel, I. I. Knyshenko, V. N. Kornoukhov, S. A. Mezentseva, I. N. Mirmov, A. I. Ostrinsky, V. V. Petukhov, A. M. Pshukov. N. Ye. Revzin, A. A. Shikhin, P. V. Timofeyev, E. P. Veretenkin, V. M. Vermul, Yu. Zakharov, G. T. Zatsepin, V. I. Zhandarov, T. J. Bowles, B. T. Cleveland, S. R. Elliott, H. A. O'Brien, D. L. Wark, J. F. Wilkerson, R. Davis, Jr., K. Lande, M. L. Cherry, and R. T. Kouzes.

14 The GALlEX Collaboration: E. Bellotti, H. Lalla, S. Pezzoni, P. Anselmann, W. Hampel, G. Heusser, J. Kiko, T. Kirsten, E. Pernicka, R. Plaga, B. Povh, C. Schlosser, H. Völk, R. Wink, M. Wojcik, R. v. Ammon, M. Balata, K. Ebert, T. Fritsch, K. Hellriegel, E. Henrich, L. Stieglitz, F. Weyrich, O. Cremonesi, E. Fiorini, S. Ragazzi, L. Zanotti, F. v. Feilitzsch, R. Mössbauer, U. Schanda, G. Berthomieu, E. Schatzman, I. Carmi, I. Dostrovsky, S. d'Angelo, C. Bacci, P. Belli, R. Bernabei, L. Paoluzi, M. Cribier, G. Dupont, L. Gosset, B. Pichard, J. Rich, M. Spiro, T. Stolarczyk, C. Tao, D. Vignaud, R. L. Hahn, F. X. Hartmann, J. K. Rowley, R. W. Stoenner, and J. Weneser.

15 A. I. Abazoo et al., preprint.

16 J. Bahcall and H. Bethe, Phys. Rev. Lett. 65 (1990) 2233.

17 J. J. Simpson, Phys. Rev. Lett. 54 (1985) 1891.

18 A. Hime and N. A. Jelley, Phys. Lett. B257 (1991) 441.

19 Baskar Sur et al., Phys. Rev. Lett. 66 (1991) 2444.

20 I. Žlimen et al., Phys. Rev. Lett. 67 (1991) 560. 
21 H. W. Becker et al., preprint. CALT-63-605.

22 D. Hetherington et al., Phys. Rev. C36 (198i) 1504.

23 J. Ashman et al.. Nucl. Phys. B328 (1989) 1.

24 J. Ellis and R. L. Jaffe, Phys. Rev. D9 (1974) 1444.

25 J. Collins, F. Wilczek, and Z. Zee, Phys. Rev. D18 (1978) 242.

26 L. A. Ahrens et al. Phys. Rev. D35 (1987) 785.

27 E. J. Beise and R. D. McKeown, Comm. on Nucl. Part. Phys. 20 (1991) 105. 

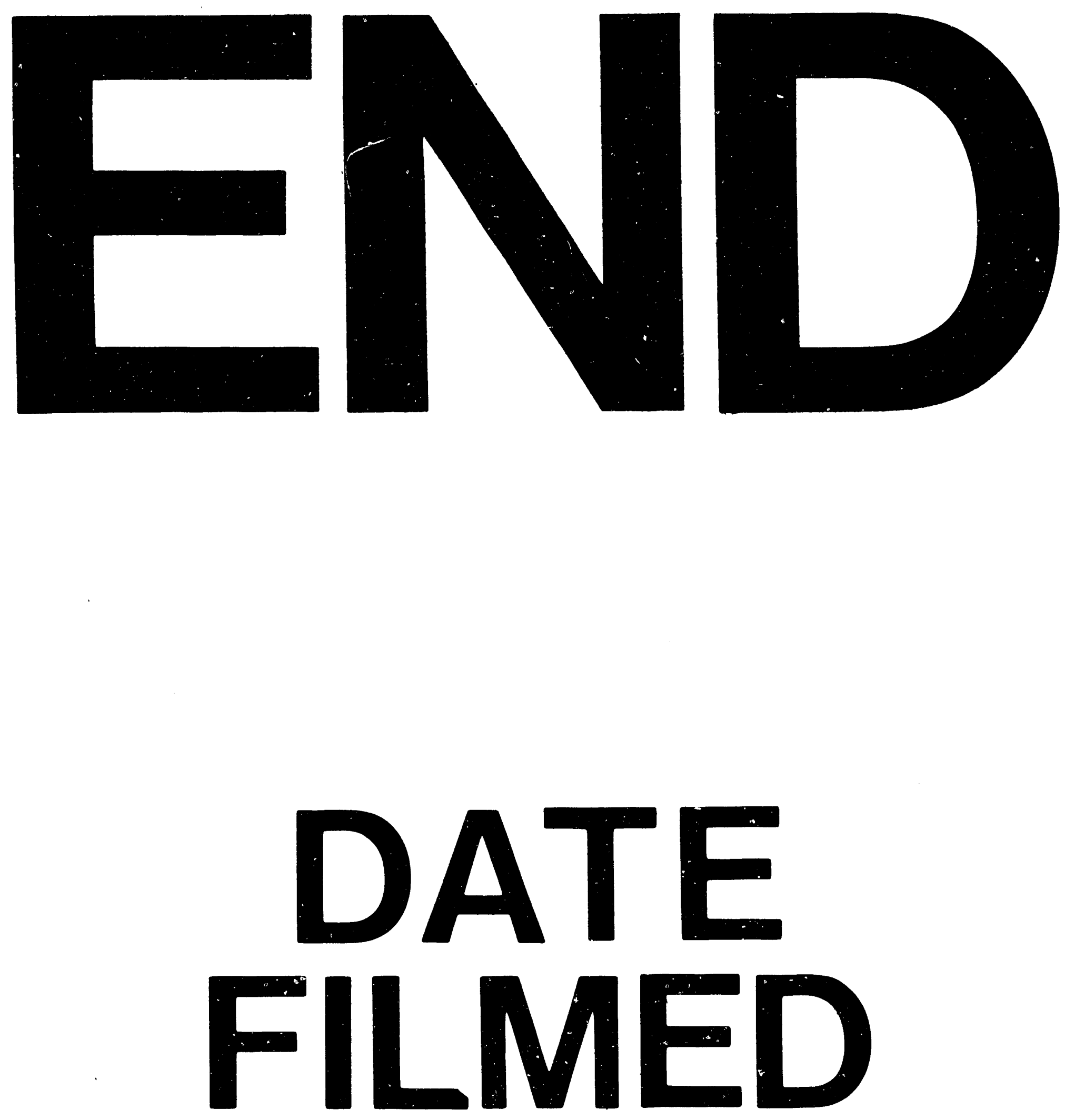

$\pm$

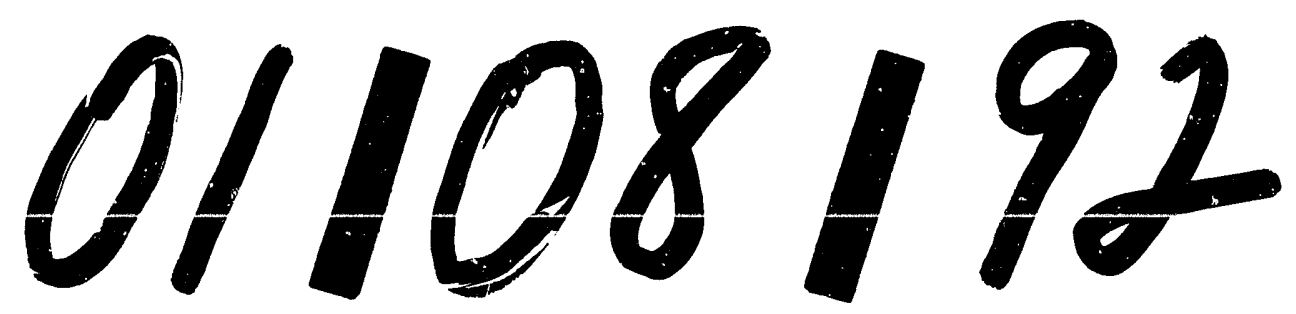


